\title{
Programmable RNA editing with compact CRISPR-Cas13 systems from uncultivated microbes
}

\author{
Chunlong Xu $\mathbf{u}^{1,9}$, Yingsi Zhou ${ }^{1,9 凶}$, Qingquan Xiao, 1,2,9, Bingbing He ${ }^{1,2,9}$, Guannan Geng ${ }^{1,9}$, \\ Zikang Wang', Birong Cao ${ }^{2,3}$, Xue Dong, ${ }^{1}$ Weiya Bai ${ }^{4}$, Yifan Wang ${ }^{1}$ Xiang Wang5 , Dongming Zhou ${ }^{5,6}$,

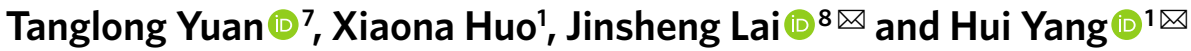

Competitive coevolution between microbes and viruses has led to the diversification of CRISPR-Cas defense systems against infectious agents. By analyzing metagenomic terabase datasets, we identified two compact families (775 to 803 amino acids (aa)) of CRISPR-Cas ribonucleases from hypersaline samples, named Cas13X and Cas13Y. We engineered Cas13X.1 (775 aa) for RNA interference experiments in mammalian cell lines. We found Cas13X.1 could tolerate single-nucleotide mismatches in RNA recognition, facilitating prophylactic RNA virus inhibition. Moreover, a minimal RNA base editor, composed of engineered deaminase (385 aa) and truncated Cas13X.1 (445 aa), exhibited robust editing efficiency and high specificity to induce RNA base conversions. Our results suggest that there exist untapped bacterial defense systems in natural microbes that can function efficiently in mammalian cells, and thus potentially are useful for RNA-editing-based research.

C RISPR-Cas systems have been classified into six subtypes and numerous orthologs in the wide spectrum of the microbial community ${ }^{1}$. Recent identification of compact CRISPR systems in uncultured microbes for type II and V families further broadens our knowledge on widespread coevolution between diverse CRISPR machinery and infectious agents ${ }^{2-4}$. Moreover, compact CRISPR effectors are highly preferred to generate CRISPR-based therapeutic modalities due to the in vivo delivery constraints of adeno-associated virus (AAV), commonly used for the treatment of durable diseases ${ }^{5}$. In contrast with the DNA-targeting activity of Cas9 and Cas12, Cas13 is a single effector recently identified in type VI CRISPR systems for RNA-guided RNA-interfering activity ${ }^{6,7}$. CRISPR-Cas13 empowers versatile applications for RNA research in both mammalian cells and plants, such as live imaging, RNA degradation, base editing and nucleic acid detection ${ }^{8}$. Numerous Cas 13 effectors divided into four families have been identified previously; however, the uncharacterized space of CRISPR-Cas13 systems in natural microbes remained elusive.

Here, we identified two compact families of CRISPR-Cas13 in metagenomic datasets and engineered them for RNA degradation and RNA base conversion in mammalian cells.

\section{Results}

Identification of type VI-X and VI-Y Cas ribonuclease families. We developed a computational pipeline (Extended Data Fig. 1a) to search for previously uncharacterized CRISPR-Cas13 systems from metagenomic datasets. Using the CRISPR array as a search anchor, we first obtained metagenomic assemblies from the JGI database and adapted existing algorithms for de novo CRISPR array detection $^{6}$. This led to the identification of 340,425 putative CRISPR repeat arrays. Up to 10 kilobases $(\mathrm{kb})$ of genomic DNA sequence flanking each CRISPR array was extracted to further identify predicted protein-coding genes in the immediate vicinity. To identify compact Cas 13 effectors, we searched among 250,901 candidate proteins with 400-900-aa residues and within 10 protein-coding genes associated with the CRISPR array, and found 24,959 proteins containing two RxxxxH motifs of the HEPN ribonuclease domain separately located at the $\mathrm{N}$ and $\mathrm{C}$ termini of the protein. Among RxxxxH motif-containing proteins, 64 contained two RxxxxH motifs of the three following types: RNxxxH, RHxxxH and RQxxxH. These three types were also found in the majority of previously known Cas13 (Extended Data Fig. 1b). Based on the fact that reported CRISPRCas13 systems have a single CRISPR RNA (crRNA) with conserved stem-loop structure ${ }^{10}$, we identified 31 Cas 13 candidates. After excluding proteins with known functions in the National Center for Biotechnology Information (NCBI) non-redundant protein (NR) database, we obtained six candidate Cas 13 proteins (Supplementary Tables 1 and 2). Further alignment of the six proteins back to the original pool of 24,959 proteins yielded one more candidate protein with RNxxxH and RxxxxH motifs. Furthermore, BLAST searches detected no sequence similarity of the identified Cas13 proteins with any of the previously identified type VI effector proteins in the NCBI NR database based on an $E$ value cutoff of $1 \times 10^{-10}$ (ref. ${ }^{11}$ ) (Supplementary Table 3). By analyzing protein sequence similarity

IInstitute of Neuroscience, State Key Laboratory of Neuroscience, Key Laboratory of Primate Neurobiology, CAS Center for Excellence in Brain Science and Intelligence Technology, Shanghai Research Center for Brain Science and Brain-Inspired Intelligence, Shanghai Institutes for Biological Sciences, Chinese Academy of Sciences, Shanghai, China. ${ }^{2}$ College of Life Sciences, University of Chinese Academy of Sciences, Beijing, China. ${ }^{3} \mathrm{CAS}$ Key Laboratory of Receptor Research, Shanghai Institute of Materia Medica, Chinese Academy of Sciences, Shanghai, China. ${ }^{4}$ Huigene Therapeutics Inc., Shanghai, China. ${ }^{5}$ Shanghai Public Health Clinical Center, Fudan University, Shanghai, China. ${ }^{6}$ Department of Pathogen Biology, School of Basic Medical Sciences, Tianjin Medical University, Tianjin, China. ${ }^{7}$ Center for Animal Genomics, Agricultural Genome Institute at Shenzhen, Chinese Academy of Agricultural Sciences, Shenzhen, China. ${ }^{8}$ State Key Laboratory of Agrobiotechnology and National Maize Improvement Center, Department of Plant Genetics and Breeding, China Agricultural University, Beijing, China. ${ }^{9}$ These authors contributed equally: Chunlong Xu, Yingsi Zhou, Qingquan Xiao, Bingbing He, Guannan Geng. 凶e-mail: yszhou@ion.ac.cn; jlai@cau.edu.cn; huiyang@ion.ac.cn 
a

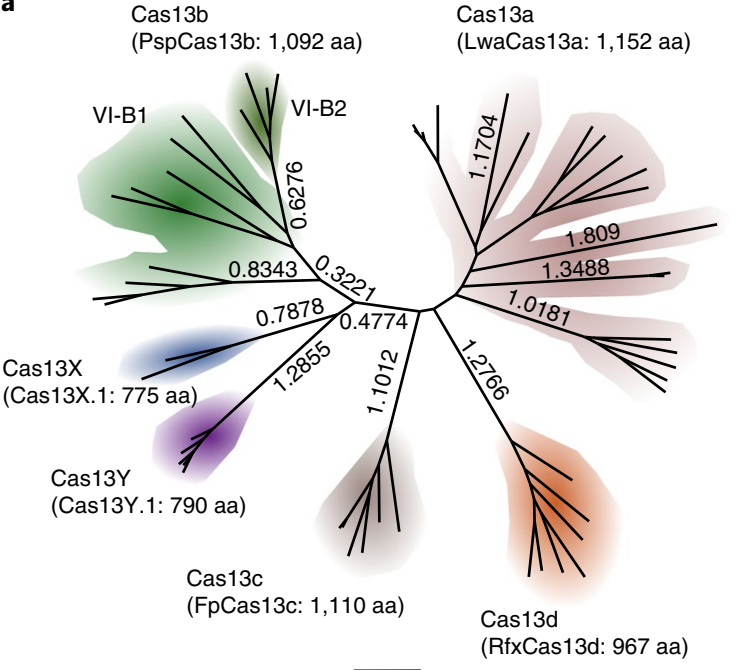

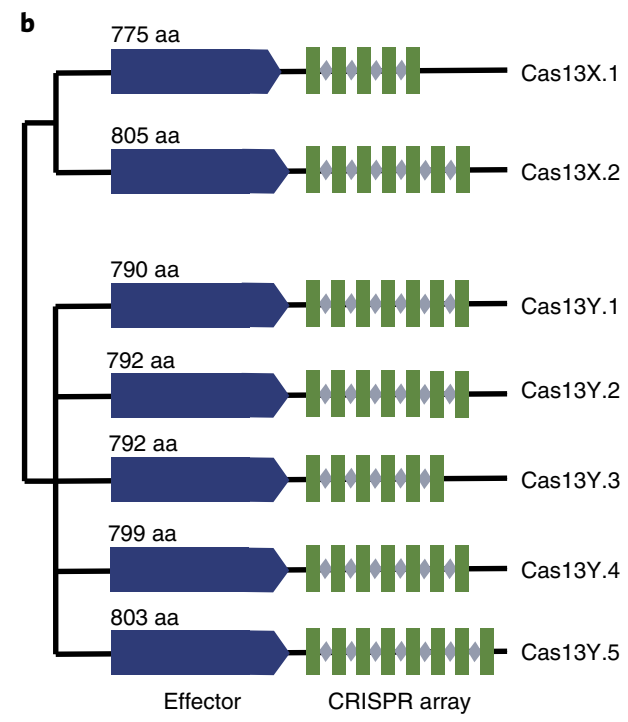

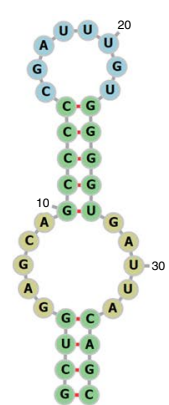

Cas13X.1

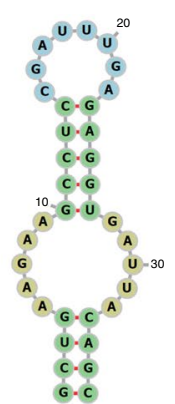

Cas13X.2

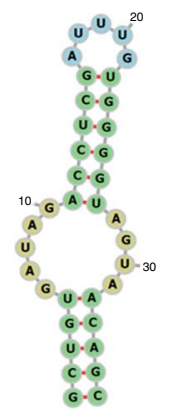

Cas13Y.1

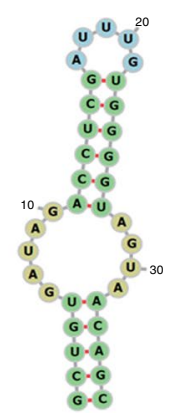

Cas13Y.2

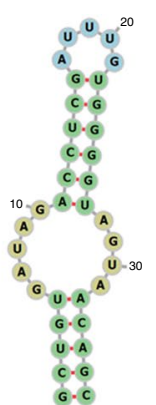

Cas13Y.3

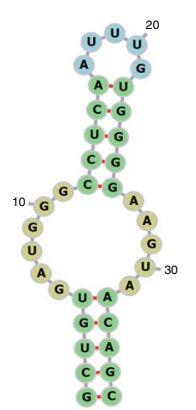

Cas13Y.4

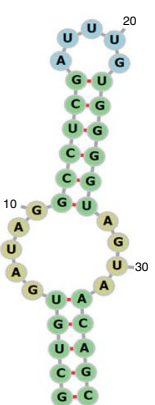

Cas13Y.5

d
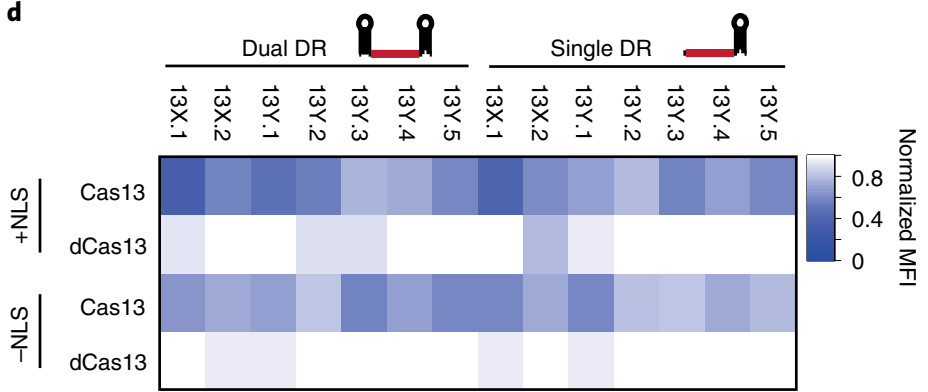

- Single-DR Dual-DR

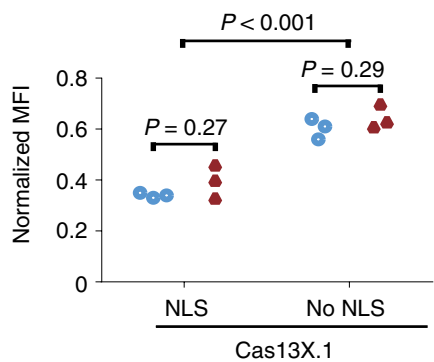

Fig. 1 | Identification and characterization of type VI-X and VI-Y CRISPR systems. a, Maximum-likelihood tree of Cas13X, Cas13Y and previously reported Cas13a (refs. ${ }^{17,32,33}$ ), Cas13b (refs. ${ }^{16,34}$ ), Cas13c and Cas13d (ref. ${ }^{15}$ ). Commonly used family members and protein sizes are shown in parentheses. The evolutionary distance scale of 0.5 is shown. $\mathbf{b}$. Maximum-likelihood phylogenetic tree of Cas13X and Cas13Y proteins identified in this study, with the full Cas13X and Cas13Y CRISPR loci drawn along with conserved HEPN RNase domains. Blue and green rectangles indicate Cas13 proteins and CRISPR DRs, respectively. Gray diamonds denote spacer sequences. c, Predicted secondary RNA structures of DR sequences for Cas $13 X$ and Cas $13 Y$ proteins. d, Heatmap of mCherry protein knockdown activity of Cas13X and Cas13Y orthologs in HEK293T cells using pre-crRNA or crRNA. e, Effect of pre-crRNA versus crRNA and NLS versus no NLS on knockdown activity of Cas13X.1. Normalized MFI, mean fluorescence intensity relative to the nontargeting condition $(n=3)$. All values shown are mean \pm s.e.m. $P$ values are by two-sided unpaired $t$-test.

among the seven Cas 13 variants, two distinct groups were found, corroborated by HEPN domain alignment results (Extended Data Fig. 1d,e and Supplementary Table 4). We therefore classify the seven proteins (size ranging from 775 to 803 aa) into two Cas 13 families, including two members ('Cas13X.1', 'Cas13X.2') in VI-X, and five members ('Cas13Y.1' to 'Cas13Y.5') in VI-Y (Fig. 1a,b).

To investigate potential targets of natural crRNA in the CRISPR loci (Supplementary Table 5), we conducted a target search in metagenomic datasets of Cas13X and Cas13Y as well as NCBI viral databases. Fifteen potential target sequences including three perfect matches were identified from metagenomic datasets for crRNA arrays associated with Cas13X.1, Cas13X.2, Cas13Y.2 and Cas13Y.4 (Supplementary Table 6). Moreover, one perfect match associated with the 'Ga0307438_1084463' contig from the Cas13f.5 sample has very high similarity $\left(E\right.$ value $\left.<1 \times 10^{-100}\right)$ with the DNA adenine methyltransferase gene previously reported to be carried by prophages in some bacteria ${ }^{12-14}$. From the GenBank-phage, RefSeq-plasmid and IMG/VR databases, four crRNAs in the array associated with Cas13X.2, Cas13Y.2 and Cas13Y.4 matched potential target sequences that have 3-5 mismatches with spacer sequences. 
These potential target sequences are from plasmids found in natural microorganisms such as Borrelia miyamotoi, Anabaena variabilis, Nostoc sp. and Acinetobacter pittii (Supplementary Table 6). It is likely that Cas13X and Cas13Y are deployed by their host to prevent invasion of these mobile genetic elements containing the target sequences.

Engineering Cas13X.1 for RNA interference in mammalian cells. To identify highly active Cas 13 orthologs, we use a eukaryotic cell-based mCherry reporter system (Extended Data Fig. 2a) to examine the RNA-targeting interference activity of the seven Cas13 proteins. By synthesizing the human-codon-optimized version of each protein, we generated mammalian expression plasmids carrying the catalytically active or inactive proteins by mutating $\mathrm{RxxxxH}$ motifs $^{7,15}$ (Extended Data Fig. 2b). Each protein was then fused with both $\mathrm{N}$ - and C-terminal nuclear localization signals (NLSs). These VI-X and VI-Y proteins were paired with two distinct forms of guide RNAs, with either a 30-nucleotide (nt) spacer flanked by two 36-nt direct repeat (DR) sequences to mimic an unprocessed guide RNA (pre-crRNA) or a 36-nt DR with a 30-nt spacer (crRNA) predicted to mimic mature guide RNAs (Fig. 1c,d). To determine crRNA architecture, we first tested DR position at the $5^{\prime}$ or $3^{\prime}$ end of crRNA with a reporter inhibition assay (Extended Data Fig. 2c). The crRNA with a $3^{\prime}$ DR instead of a $5^{\prime}$ DR showed substantial suppression of reporter expression (Extended Data Fig. 2d), indicating that the crRNA accompanying Cas13X.1 shared a similar 3' DR structure with that of previously reported Cas13b (ref. ${ }^{16}$ ). We then assessed the abilities of different VI-X and VI-Y proteins to knock down the mCherry reporter level in HEK293T cells. At $2 \mathrm{~d}$ after transfection with the plasmids expressing each of the VI-X and VI-Y proteins and the corresponding single target-specific crRNA, we observed significant reduction of mCherry protein, with Cas13X.1 exhibiting the highest knockdown efficiency (Fig. 1d,e). In contrast, transfection with nontargeting crRNA together with each Cas13, or, alternatively, crRNA with inactive Cas13, had no significant effect on the mCherry level (Fig. 1d,e and Extended Data Fig. 2d), suggesting crRNA- and HEPN-dependent knockdown. We also found that both the single DR crRNA and pre-crRNA with dual DR could mediate potent knockdown, and NLS significantly improved knockdown activity of Cas13X.1 (Fig. 1d,e). To determine the optimal spacer length for efficient Cas13X.1 targeting, we targeted mCherry with crRNA-carrying spacers of different lengths ranging from 5 to $50 \mathrm{nt}$, varying with a step of $1 \mathrm{nt}$ between 15 and $30 \mathrm{nt}$ or with step of $5 \mathrm{nt}$ for the rest of the lengths (Extended Data Fig. 2e). We found reporter inhibition activity to be robustly efficient at all three mCherry-targeting loci when using a 30-nt spacer; this length was consistent with the finding in the Cas13X-associated CRISPR array of the uncultured microorganism (Extended Data Fig. 2e). Furthermore, $15 \mathrm{nt}$ was determined as the minimal length for the spacer to mediate detectable knockdown in HEK293T cells. Thus, crRNAs with a 30-nt spacer were used for the following RNA interference experiments unless otherwise indicated. To investigate any protospacer flanking sequence (PFS) requirements for Cas13X.1, we carried out the PFS screening analysis and found no PFS bias in Cas13X.1 for efficient RNA knockdown activity (Extended Data Fig. 2f).

We next sought to compare the knockdown efficiency of Cas13X.1 and Cas13Y.1 against that of previously identified Cas13 proteins, Cas13a (ref. ${ }^{17}$ ), Cas13b (ref. ${ }^{16}$ ) and Cas13d (ref. ${ }^{15}$ ). Across three target loci in $m$ Cherry, Cas13X.1, Cas13Y.1 and RfxCas13d overall outperformed LwaCas13a and PspCas13b in HEK293T cells at $48 \mathrm{~h}$ after transfection (Fig. 2a and Extended Data Fig. 3c). To confirm that RNA interference by Cas13X.1 and Cas13Y.1 is broadly applicable as previously reported Cas $13 \mathrm{a} / \mathrm{b} / \mathrm{d}$, we selected a panel of 12 additional human genes with diverse roles in mammalian cells, using one crRNA per gene. Cas13X.1, Cas13Y.1 and
RfxCas13d showed comparable or higher knockdown efficiency than LwaCas13a and PspCas13b (Fig. 2b and Extended Data Fig. 3d). Moreover, we designed two additional crRNAs on the same 12 genes for Cas13X.1 and found that Cas13X.1 consistently showed high-level knockdown activity for each gene, using any of the three crRNAs (Extended Data Fig. 3a,b), indicating the uniformity of the Cas13X.1 system for RNA interference. Because the Cas13 family is capable of processing its own CRISPR array ${ }^{15}$, we next leveraged this property for the delivery of pre-crRNA for multiple targeting with a simple single-vector system (Fig. 2c). We found that robust simultaneous knockdown of four RNA transcripts could be achieved by transfection of Cas13X.1 together with an array encoding four crRNAs, each tiling one mRNA (EZH2, HRAS or PPARG) and a nuclear-localized long noncoding RNA (MALAT1) (Fig. 2c). Furthermore, we performed transcriptome-wide RNA-sequencing (RNA-seq) analysis on Cas13X.1 targeting B4GALNT1 and EZH2 genes in HEK293T cells. It was found that Cas13X.1 induced a comparable number of differentially expressed genes with RfxCas13d after knockdown of B4GALNT1 and EZH2 (Fig. 2d). Among the 48 downregulated genes in the Cas13X-mediated $E Z H 2$ knockdown experiment, 9 affected downregulated genes were reported to be regulated by EZH2 (Supplementary Tables 7-10). We further made a genome-wide search for similar sequences with a crRNA target from both B4GALNT1 and EZH2 genes, and found that the most similar sequences had at least 6 mismatches with crRNA targets. All predicted off-target genes were also nonsignificantly regulated for Cas13X.1 (Extended Data Fig. 4). To examine the target-dependent collateral ribonuclease activity of Cas13X.1 with previously compared RfxCas13d and LwaCas13a, we used EGFP-transgenic HEK293T cells to monitor EGFP expression as an indicator of the potential collateral effect in mammalian cells (Extended Data Fig. 5a) when targeting transiently overexpressed mCherry and endogenously expressed genes. It was found that comparably low collateral activities for EGFP were found for LwaCas13a, RfxCas13d and Cas13X.1 when transiently overexpressing the mCherry target in HEK293T (Extended Data Fig. 5b); but, for endogenous RNA knockdown, collateral effects for three different Cas13 proteins were undetectable on three target genes by the reporter assay, which agrees with a previous study ${ }^{18}$ (Extended Data Fig. $5 c-e$ ). Furthermore, we conducted a fluorophore-quencher assay, in which cleavage of dye-labeled single-stranded RNA (ssRNA) generates a fluorescent signal, and found that Cas13X.1 exhibited lower in vitro collateral RNase activity than RfxCas13d and LwaCas13a (Extended Data Fig. 6a,b). Taken together, these results indicate that Cas13X.1 offers a compact RNA interference tool with relatively high efficiency and specificity.

Antiviral activity of Cas13X.1 in mammalian cells. CRISPRCas13, as a naturally evolved antiviral system, could potentially be developed as an antivirus modality for combating human infections ${ }^{19-21}$. Therefore, we investigated whether Cas13X.1, with smaller size and comparable efficiency to previously identified RfxCas13d, could be used for prophylactic RNA virus inhibition to complement the current Cas13 toolbox. To create effective and specific crRNA sequences to target and cleave SARS-CoV-2, we first performed a bioinformatics analysis by aligning published SARS-CoV-2 genomes ${ }^{22}$ and selected 30 crRNAs targeting RNA sites coding for RdRP (RNA-dependent RNA polymerase) and E (envelope) proteins (with $15 \mathrm{crRNAs}$ for each). Proof-of-concept experiments were performed on RdRP and E sequences which are conserved among SARS-CoV viruses (Fig. 3a). The RdRP protein is the antiviral target for remdesivir ${ }^{23}$ and the $\mathrm{E}$ protein is critical for SARS-CoV pathogenesis ${ }^{24}$. To evaluate whether Cas13X.1 is effective for degrading SARS-CoV-2 sequences, we created a reporter by fusing GFP with synthesized partial SARS-CoV-2 fragments of RdRP (genome coordinates 15,037-15,158 base pairs 

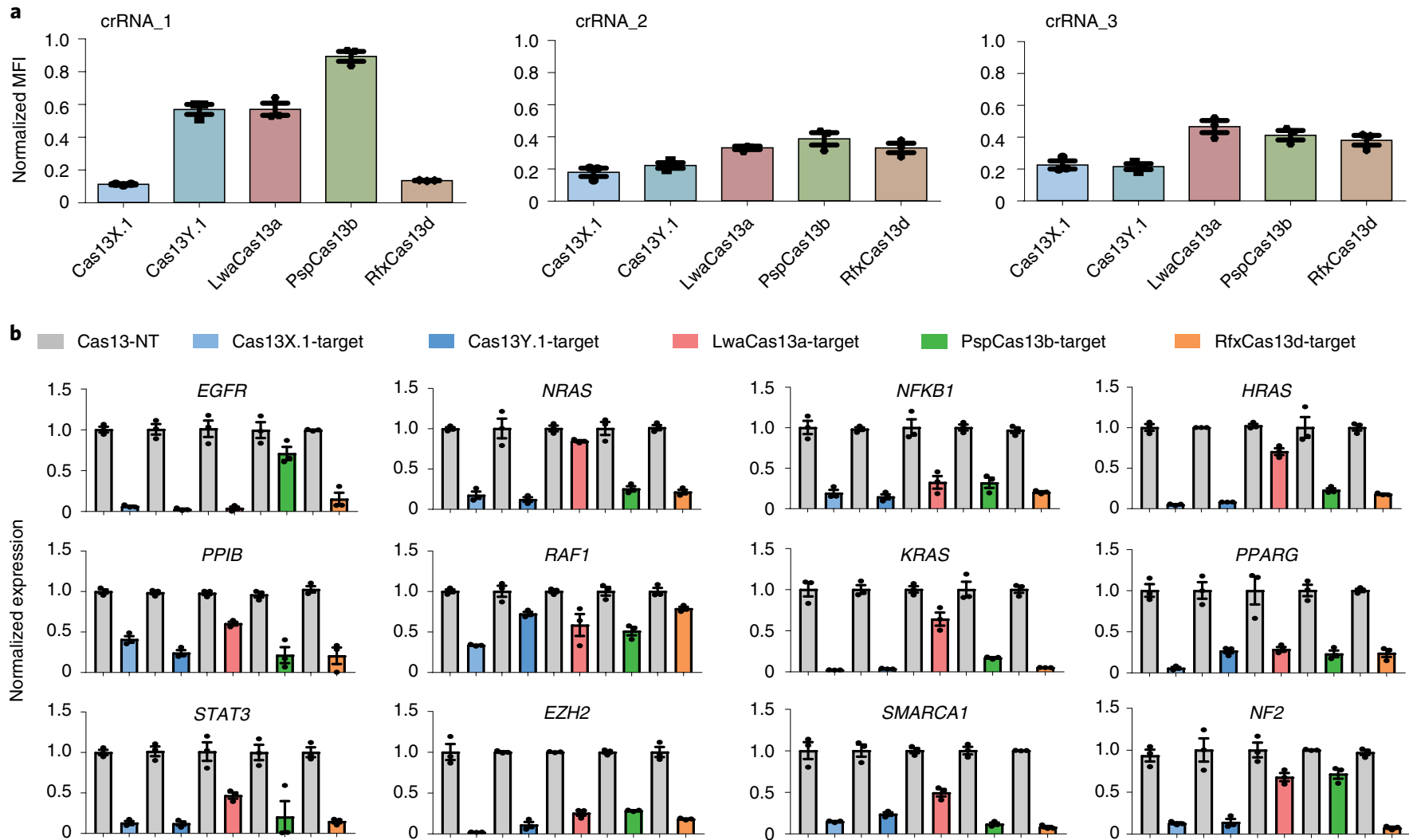

LwaCas13a-target

PspCas13b-target

RfxCas13d-target
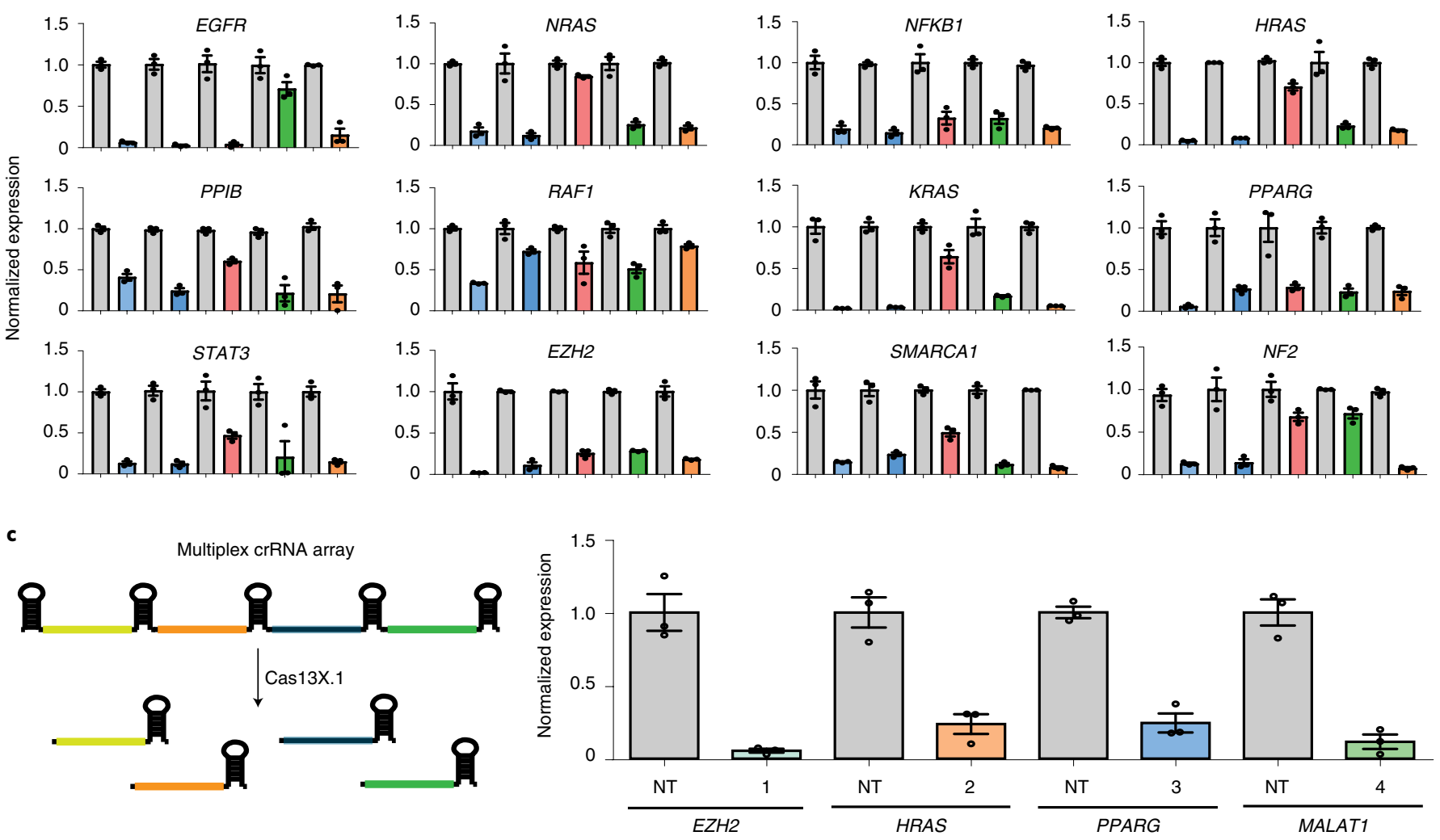

d

B4GALNT1
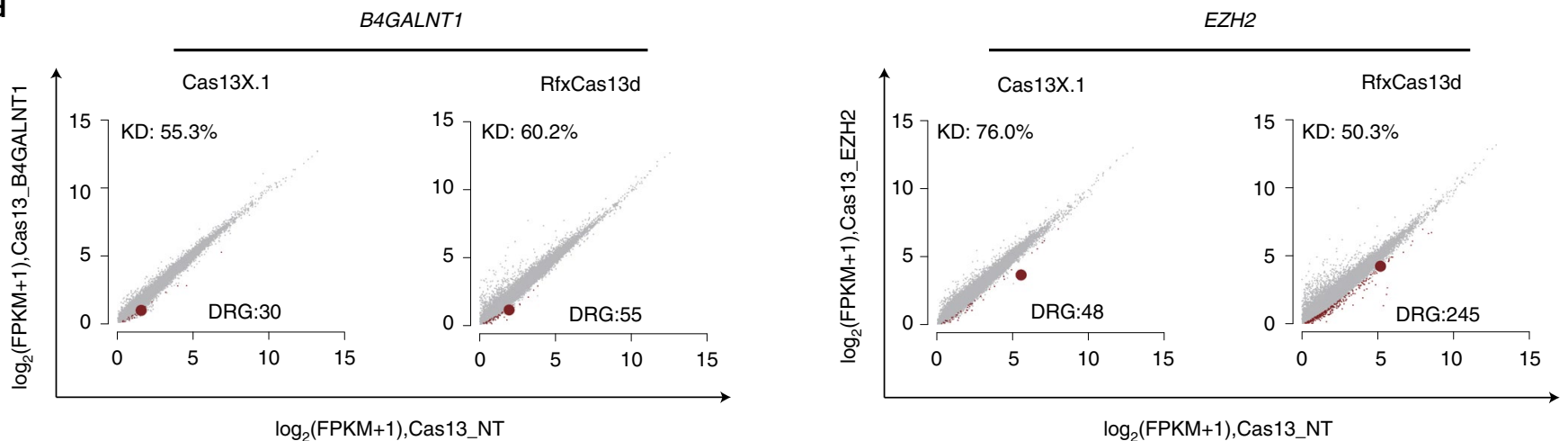

Fig. 2 | Efficient and specific interference activity of Cas13X.1 against transcripts in HEK293 cells. a, Reporter inhibition assay results of comparing activity among Cas13X.1, Cas13Y.1, LwaCas13a, PspCas13b and RfxCas13d for three different mCherry-targeting crRNAs in HEK293T. Normalized MFI, mean fluorescence intensity relative to the nontargeting condition. b. Comparison of knockdown efficiency for 12 endogenous transcripts by Cas $13 X .1$, Cas13Y.1, LwaCas13a, PspCas13b and RfxCas13d, each with one guide and a nontargeting crRNA in HEK293T cells. c, Arrays of four guides; each mediates target knockdown by Cas13X.1 in HEK293T cells via transient transfection. d, Differential gene expression analysis of RNA-seq for B4GALNT1 and EZH2 knockdown in HEK293T cells by Cas13X.1 and RfxCas13d (three biological replicates). Knockdown relative to an NT crRNA was determined by qPCR. DRG, downregulated genes; KD, knockdown; NT, nontargeting crRNA. All values shown are mean \pm s.e.m $(n=3)$. 
a

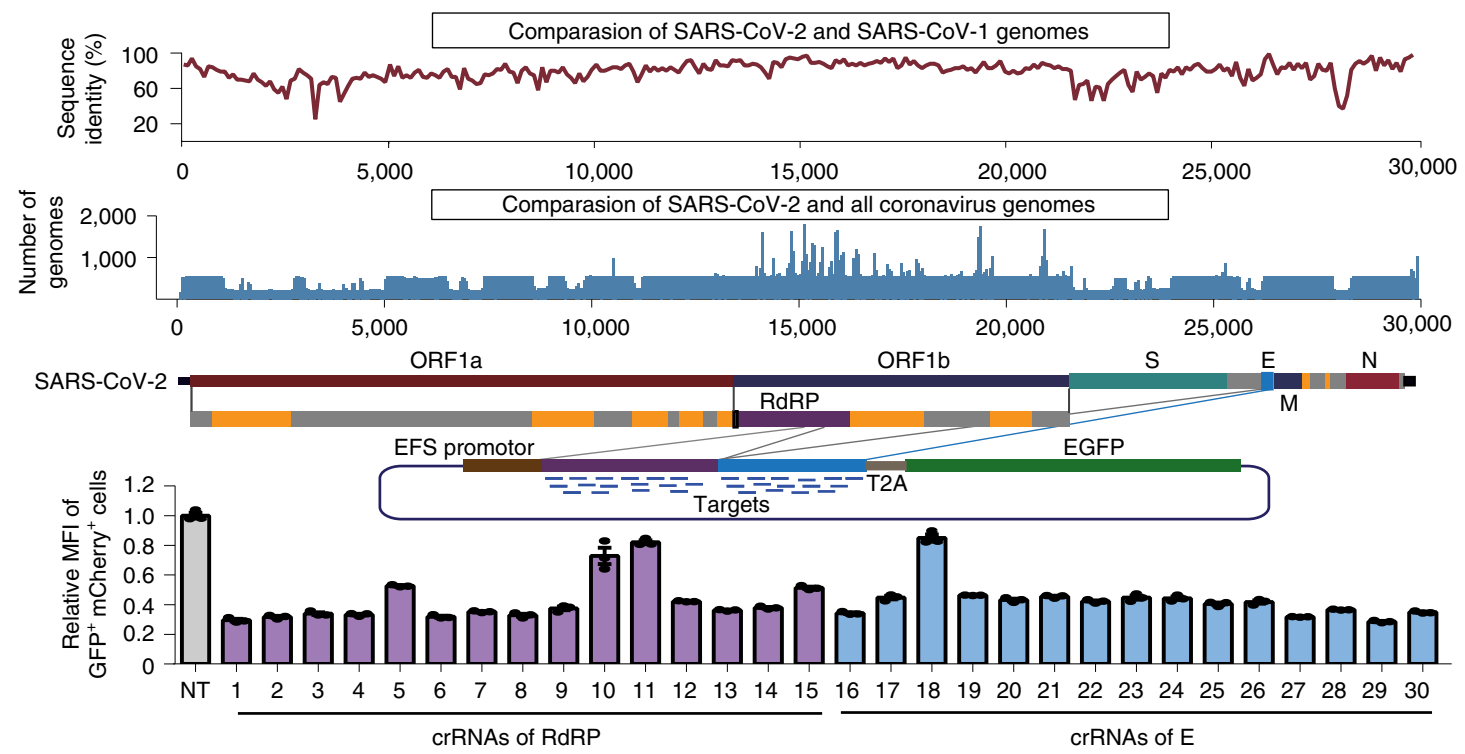

b
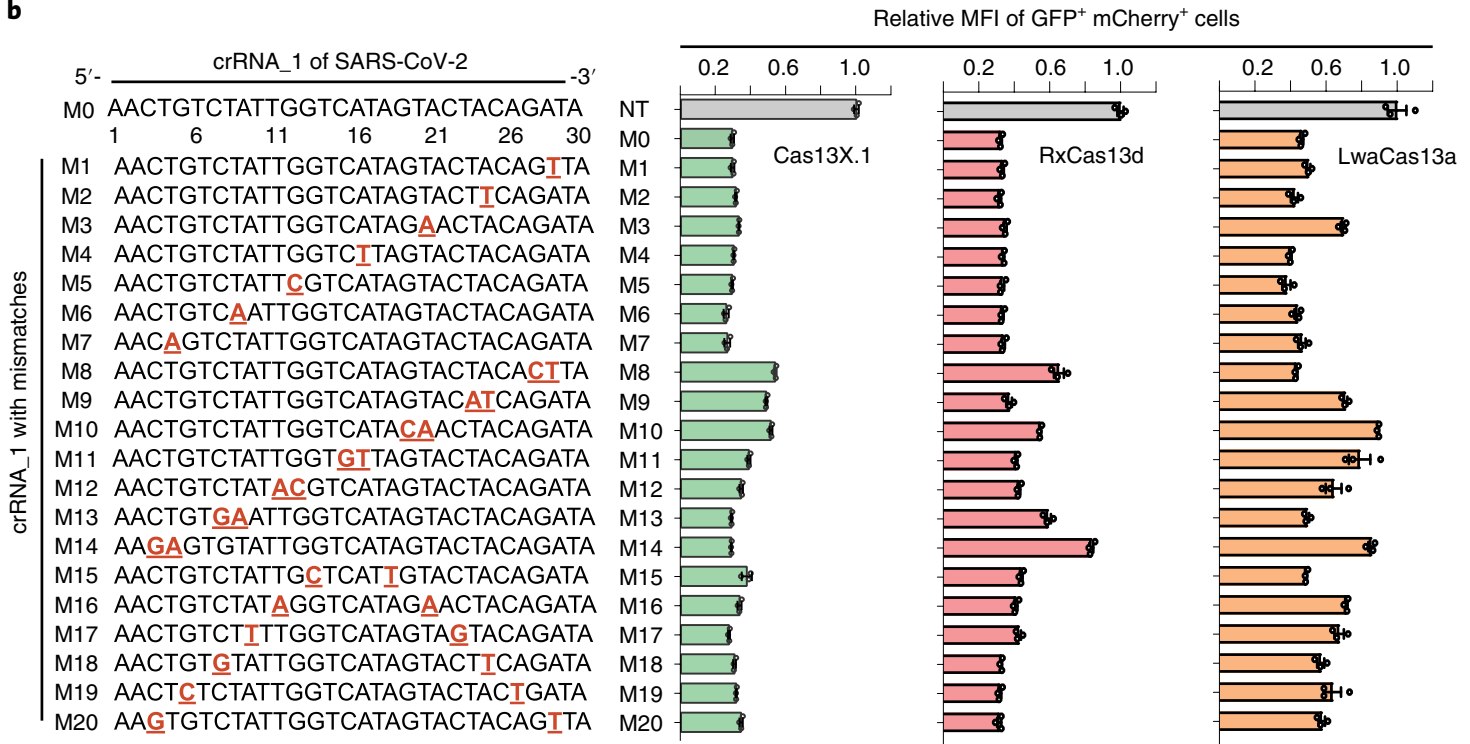

c
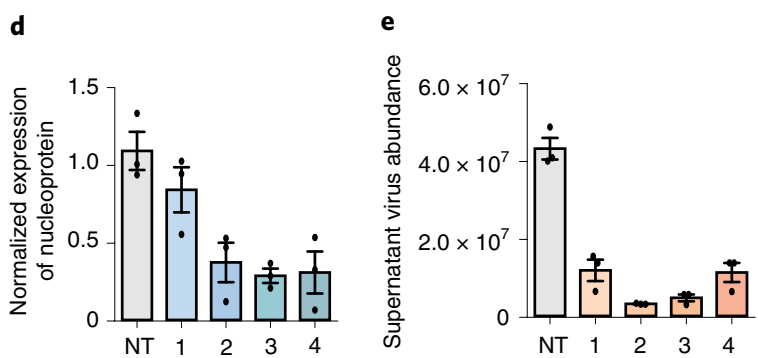

Fig. 3 Antiviral activity of Cas13X.1 in mammalian cells and mismatch tolerance features of Cas13X.1, RfxCas13d and LwaCas13a. a, Top, comparison of sequence identity between SARS-CoV-2 and SARS-CoV-1 genomes, and alignment comparison of SARS-CoV-2 and all coronavirus genomes. Middle, schematic diagram of the reporter consists of EFS promoter, GFP and the synthesized RdRP and E fragment sequences. Bottom, GFP expression after cotransfection of the reporter and Cas13X.1/crRNA, as measured by flow cytometry. Mean GFP fluorescence intensity changes of the reporter caused by 30 different targeting crRNAs, relative to nontargeting (NT) crRNA. b, Left, crRNA nucleotide identity or mismatch types (single-nucleotide mismatch or two tandem-nucleotide mismatches). Right, changes of GFP fluorescence intensity caused by cotransfection of reporter, Cas13X.1, RfxCas13d or LwaCas13a together with each of 20 different versions of crRNA_1 with mismatched mutations, relative to NT crRNA. c, Procedure for testing Cas13X.1-mediated anti-H1N1 activity in H1N1 IAV-infected MDCK cells. MDCK cells were first transfected with Cas13X.1/crRNA vectors and later challenged with H1N1 IAV. Supernatant was collected to analyze IAV abundance after $48 \mathrm{~h}$ of IAV infection. d, IAV RNA knockdown efficiency resulting from transfection of nucleoprotein (NP)-targeting Cas13X.1/crRNA. Transcript levels are relative to NT crRNA control. e, Changes of IAV abundance following Cas13X.1/crRNA transfection, analyzed by absolute RT-qPCR of supernatant from infected cultures. All values in $\mathbf{a}, \mathbf{b}, \mathbf{d}$ and $\mathbf{e}$ are shown as mean \pm s.e.m $(n=3)$. IAV, influenza A virus; M, membrane protein; N, nucleocapsid protein; S, spike protein. 
a
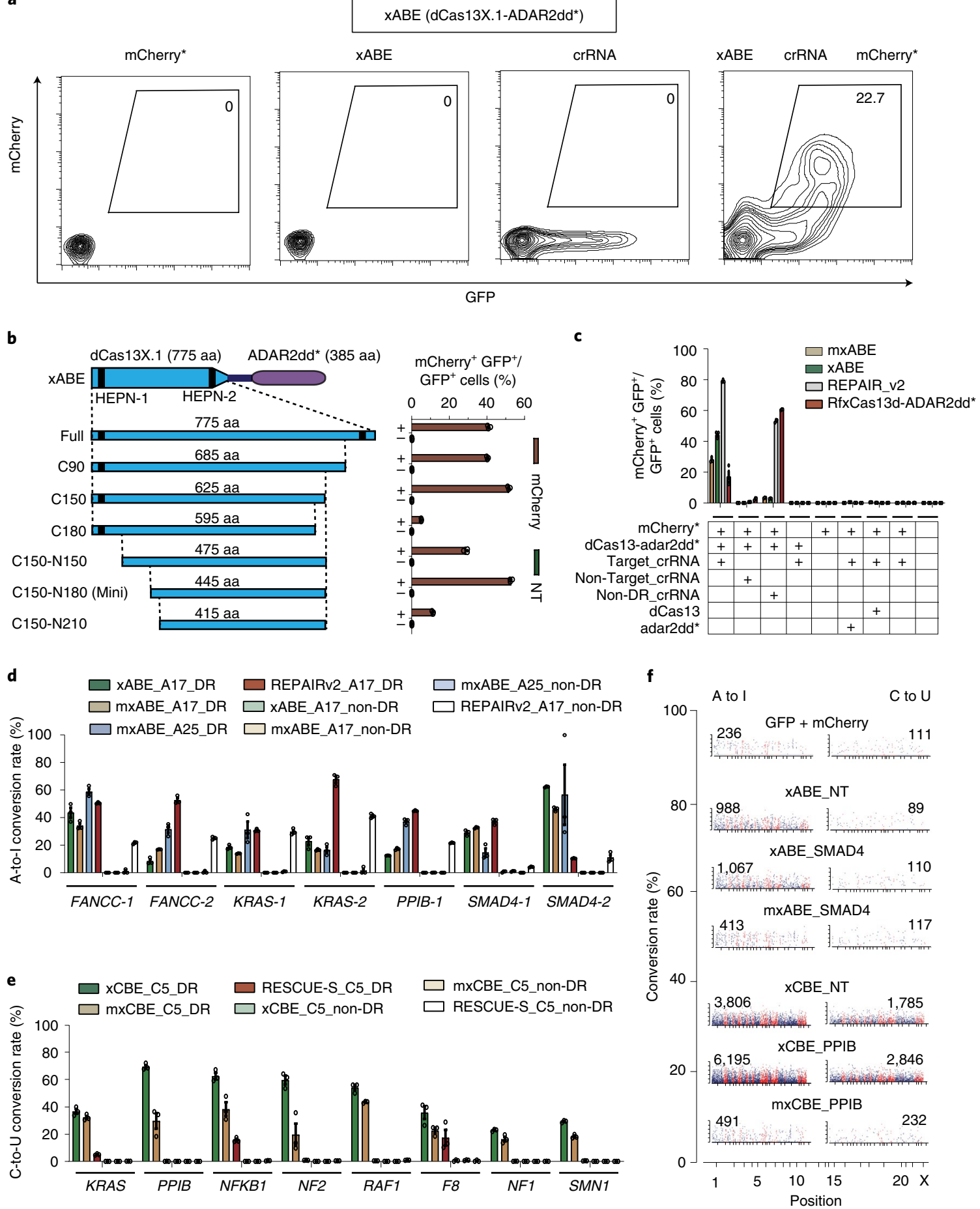

Fig. 4 | Truncated dCas13X.1 with ADAR2dd variants for efficient RNA base editing. a, Restored expression of mutant reporter by xABE RNA base editor as measured with flow cytometry. $\mathbf{b}$, The activity of a variety of truncated $X A B E$ variants was analyzed by reporter assay. The black bar on Cas13X.1 indicates the HEPN domain. c, Reporter assay shows A-to-I editing activity among various editors including mxABE, XABE, REPAIR_v2 and RfxCas13d-ADAR2dd ${ }^{\star}$. d, A-to-I editing efficiency of $x A B E, m \times A B E$ and REPAIR_v2 on endogenous transcripts in HEK293T analyzed with deep sequencing. e, $\mathrm{C}$-to- $U$ editing efficiency of $x \mathrm{CBE}, \mathrm{mxCBE}$ and RESCUE_S on endogenous transcripts in HEK293T analyzed with deep sequencing. $\mathbf{f}$, Manhattan plots of transcriptome-wide off-target RNA editing analysis for GFP/mCherry (control), $x A B E$, $m \times A B E, \times C B E$ and mxCBE transfection experiments in HEK293T cells (A-to-I editor targeting endogenous SMAD4 RNA; C-to-U editor targeting endogenous PPIB RNA). The $x$ and $y$ axes are proportionally enlarged with each Manhattan plot to make the axis legend clear. Non-DR, guide RNA without DRs; NT, nontargeting crRNA. All values are presented as mean \pm s.e.m $(n=3)$.

(bp)) and E (26,232-26,394bp) (Fig. 3a). At $48 \mathrm{~h}$ after cotransfection of HEK293T cells with the reporter and Cas13X.1/crRNAs, we observed that nearly all RdRP- and E-targeting crRNAs tested (27 out of 30) were able to support the suppression of GFP fluorescence in the cells by about $70 \%$, as compared with that found for control transfection with the nontargeting crRNA (Fig. 3a). 
We next examined the minimal number of crRNAs required to target the majority of known coronaviruses found in both humans and animals, using a similar strategy to that previously described ${ }^{19}$. From all known 3,137 coronavirus genomes, we identified approximately 7.1 million potential unique crRNA targets (Extended Data Fig. 7a). We estimated that only five 22-nt and six 30-nt crRNAs with zero mismatch were able to target over $90 \%$ of coronavirus genomes (Extended Data Fig. 7b,c). We next examined whether Cas13 could tolerate mismatches between the crRNA and the target viral RNA, enabling inhibition of more coronavirus variants without increasing crRNA number and prevention of virus escaping via mutagenesis. Thus, we assessed the knockdown activity for an example crRNA (SARS-CoV-2 crRNA_1) with one or two mismatches (Fig. 3b), and found that both Cas13X.1 and RfxCas13d could well tolerate a single-nucleotide mismatch at different positions on the example crRNA (Fig. 3b). Results on two tandem mismatches revealed a critical (seed) region between 16 and 30-nt of the crRNA for efficient Cas13X.1-induced knockdown (Fig. 3b). With the tolerance for single-nucleotide mismatch, we estimated that 3,10 and 17 crRNAs could target $95.3 \%, 99.1 \%$ and $100 \%$ of all coronaviruses, respectively (Extended Data Fig. 7d).

Next, we applied the CRISPR-Cas13X.1 strategy for inhibiting influenza RNA virus $\mathrm{H} 1 \mathrm{~N} 1$ which has a tropism for respiratory tract epithelial cells similar to SARS-CoV-2. We directly designed four crRNAs targeting at the nucleoprotein segment of the H1N1 genome which is essential for viral replication and transcription ${ }^{20,24}$. To test the antiviral ability of Cas $13 \mathrm{X} .1$ in a setting that mimics virus infection, we used influenza H1N1 strain 'A/Puerto Rico/8/1934' (ref. ${ }^{25}$ ) in the Madin-Darby canine kidney (MDCK) cell line (Fig. 3c). Compared with nontargeting crRNA, three out of four crRNAs showed high knockdown efficiency on the nucleoprotein transcript (Fig. 3d). Consistently, target-specific crRNAs significantly reduced the abundance of nucleoprotein-positive $\mathrm{H} 1 \mathrm{~N} 1$ virus found in the supernatant of infected cultures, indicating effective inhibition of viral growth (Fig. 3e). Together, these results showed that the Cas13X.1 system could be used to confer antiviral ability for mammalian cells.

Truncated dCas13X.1 with ADAR2dd variants for efficient RNA base conversions. Base editing at the RNA level by RNA-guided Cas13 enables reversible nucleotide exchange with broad applicability in biomedical research and treatment of genetic diseases. However, previous Cas13b or Cas13d was too large after fusion with ADAR2 (adenosine deaminase acting on RNA type 2) to be widely used for in vivo viral delivery. Therefore, we first fused dCas13X.1 with high-fidelity ADAR2dd (with E488Q/T375G, referred as ADAR2dd*) to generate A-to-I RNA base editors (termed ' $\mathrm{xABE}$ '). To test the activity of $\mathrm{xABE}$, we generated an RNA-editing reporter using a mutated mCherry with a nonsense mutation (W98X (UGG to UAG)), which could functionally be repaired to the wild-type codon through A-to-I editing, and mCherry fluorescence could be detected after editing (Extended Data Fig. 8a). We found that xABE effectively induced mCherry fluorescence in cells transfected with mutant mCherry transcripts, together with both $\mathrm{xABE}$ and $50-\mathrm{nt}$ crRNA, but not with either alone (Fig. 4a). To reduce the size of dCas13X.1 for efficient in vivo delivery, we generated various base editors by fusing the truncated dCas13X.1 (using a structure-guided method) with ADAR2dd* (Fig. 4b and Extended Data Fig. 8b). To this end, we systematically screened the editing activity of a variety of fused base editors with different dCas13X.1 truncations at either or both $\mathrm{N}$ and $\mathrm{C}$ termini (Fig. 4b), and identified the miniature and functional editor ('mini') with 150-aa and 180-aa truncation at C and $\mathrm{N}$ termini, respectively (Fig. $4 \mathrm{~b}$ ), suitable for packaging into commonly used AAV. We then examined the effect of mismatched base position within a 50-nt spacer on A-to-I editing efficiency for both full-size (xABE) and mini xABE (mxABE) (Extended Data
Fig. 9a), and found that mismatched base position from 15 to $25 \mathrm{nt}$ on the crRNA sequence yielded higher editing efficiency than other positions (Extended Data Fig. 9b). Furthermore, we compared xABE $(1,195 \mathrm{aa})$ and mxABE (865 aa) with dCas13b-ADAR2dd ${ }^{*}$ (REPAIR, 1,388 aa) and CasRx-ADAR2dd ${ }^{*}(1,375 \mathrm{aa})$ using crRNA with or without DR, and found only $x A B E / m x A B E$ achieved efficient crRNA-guided editing (Fig. 4c). By contrast, both REPAIR and CasRx-ADAR2 $\mathrm{dd}^{*}$ generated substantial editing with DR-free guide sequence, indicating editing mostly via a crRNA-independent pathway (Fig. 4c). Next, the RNA-editing efficiency of the full-size and mini mxABE systems was further examined in mammalian cells for several endogenous transcripts in comparison with REPAIR. We found crRNA-dependent A-to-I conversions were efficiently achieved by $\mathrm{xABE} / \mathrm{mxABE}$ editors but not REPAIR, confirming the results with reporter assay (Fig. $4 \mathrm{~d}$ and Extended Data Fig. 9c). To extend the base-editing capability of the dCas13X.1 protein, we further generated a C-to-U base editor ('xCBE') by fusing full-length or truncated dCas13X.1 with RNA cytosine deaminase derived from evolved ADAR2 (ref. ${ }^{26}$ ), and found both full-length and mini $\mathrm{xCBE}(\mathrm{mxCBE})$ could achieve more efficient C-to-U editing than the previously reported RESCUE-S (1,495 aa) system in HEK293T cells (Fig. 4e). Moreover, both truncated mxABE and mxCBE exhibited transcriptome-wide high-fidelity activity and reduced RNA off-target edits to the base level in contrast with those of full-length xABE/xCBE and REPAIR by RNA-seq analysis (Fig. 4f and Extended Data Fig. 10a,b). Therefore, mxABE and mxCBE demonstrated great potential as compact, efficient and safe RNA base editors to facilitate an AAV-based method for treating genetic diseases.

\section{Discussion}

Here, we identified two families of compact CRISPR-Cas13 systems (type VI-X and VI-Y) by mining metagenomic sequence datasets of natural uncultivated microbes, highlighting the diversity of natural microbial CRISPR systems. Given the sequence and structural differences in Cas 13 subtypes and their crRNA DR regions, Cas13 proteins might evolve to have different activity towards the same target. Thus, it is worthwhile to enrich the Cas 13 toolbox by computational mining of more metagenomic sequences generated by samples from diverse environments. In addition, we found the identified Cas13s could induce collateral cleavage of RNA in cells by targeting exogenous overexpressed genes, which is consistent with previous studies ${ }^{7,15,16,27-30}$. Although collateral activity was undetectable for Cas13 proteins when transiently targeting endogenous genes, a more sensitive way to evaluate collateral effects in mammalian cells and further long-term safety evaluation are needed for future therapeutic applications. Notably, all known Cas13 orthologs from the type VI-X and VI-Y families originated from microorganisms living in hypersaline habitats, reducing the risk of preexisting immunity found in Cas9 and Cas12 orthologs identified from pathogenic bacteria samples closely related to human ${ }^{31}$. Furthermore, by structure-guided engineering, we successfully truncated Cas13X.1 from 775 to 445 aa to generate a minimal RNA base editor for A-to-I or C-to- $\mathrm{U}$ editing on various RNA loci in mammalian cells, overcoming the in vivo delivery obstacle of various large Cas13-based base editors. We envision that these RNA editors with the compact Cas13X.1 will be useful for in vivo RNA editing-based research and therapeutic applications $\mathrm{s}^{27-30}$.

\section{Online content}

Any methods, additional references, Nature Research reporting summaries, source data, extended data, supplementary information, acknowledgements, peer review information; details of author contributions and competing interests; and statements of data and code availability are available at https://doi.org/10.1038/ s41592-021-01124-4. 
Received: 14 May 2020; Accepted: 18 March 2021;

Published online: 3 May 2021

\section{References}

1. Makarova, K. S. et al. An updated evolutionary classification of CRISPR-Cas systems. Nat. Rev. Microbiol. 13, 722-736 (2015).

2. Fonfara, I. et al. Phylogeny of Cas 9 determines functional exchangeability of dual-RNA and Cas9 among orthologous type II CRISPR-Cas systems. Nucleic Acids Res. 42, 2577-2590 (2014).

3. Harrington, L. B. et al. Programmed DNA destruction by miniature CRISPR-Cas14 enzymes. Science 362, 839-842 (2018).

4. Pausch, P. et al. CRISPR-Cas $\Phi$ from huge phages is a hypercompact genome editor. Science 369, 333-337 (2020).

5. Wang, D., Zhang, F. \& Gao, G. CRISPR-based therapeutic genome editing: strategies and in vivo delivery by AAV vectors. Cell 181, 136-150 (2020).

6. Shmakov, S. et al. Discovery and functional characterization of diverse class 2 CRISPR-Cas systems. Mol. Cell 60, 385-397 (2015).

7. Abudayyeh, O. O. et al. C2c2 is a single-component programmable RNA-guided RNA-targeting CRISPR effector. Science 353, aaf5573 (2016).

8. Smargon, A. A., Shi, Y. J. \& Yeo, G. W. RNA-targeting CRISPR systems from metagenomic discovery to transcriptomic engineering. Nat. Cell Biol. 22, 143-150 (2020).

9. Markowitz, V. M. et al. IMG: the integrated microbial genomes database and comparative analysis system. Nucleic Acids Res. 40, D115-D122 (2012).

10. O'Connell, M. R. Molecular mechanisms of RNA targeting by Cas13containing type VI CRISPR-Cas systems. J. Mol. Biol. 431, 66-87 (2019).

11. Makarova, K. S. et al. Evolutionary classification of CRISPR-Cas systems: a burst of class 2 and derived variants. Nat. Rev. Microbiol. 18, 67-83 (2020).

12. Lynch, K. H., Stothard, P. \& Dennis, J. J. Genomic analysis and relatedness of P2-like phages of the Burkholderia cepacia complex. BMC Genomics 11, 599 (2010).

13. Saridaki, A. et al. Wolbachia prophage DNA adenine methyltransferase genes in different Drosophila-Wolbachia associations. PLoS ONE 6, e19708 (2011).

14. Sternberg, N. \& Coulby, J. Cleavage of the bacteriophage P1 packaging site (pac) is regulated by adenine methylation. Proc. Natl Acad. Sci. USA 87, 8070-8074 (1990)

15. Konermann, S. et al. Transcriptome engineering with RNA-targeting type VI-D CRISPR effectors. Cell 173, 665-676.e614 (2018).

16. Smargon, A. A. et al. Cas13b is a type VI-B CRISPR-associated RNA-guided RNase differentially regulated by accessory proteins Csx27 and Csx28. Mol. Cell 65, 618-630.e617 (2017).

17. Abudayyeh, O. O. et al. RNA targeting with CRISPR-Cas13. Nature 550, 280-284 (2017).

18. Wang, Q. et al. The CRISPR-Cas13a gene-editing system induces collateral cleavage of RNA in glioma cells. Adv. Sci. (Weinh.) 6, 1901299 (2019).
19. Abbott, T. R. et al. Development of CRISPR as an antiviral strategy to combat SARS-CoV-2 and influenza. Cell https://doi.org/10.1016/j.cell.2020.04.020 (2020).

20. Freije, C. A. et al. Programmable inhibition and detection of RNA viruses using Cas13. Mol. Cell 76, 826-837.e811 (2019).

21. Nguyen, T. M., Zhang, Y. \& Pandolfi, P. P. Virus against virus: a potential treatment for 2019-nCov (SARS-CoV-2) and other RNA viruses. Cell Res. 30, 189-190 (2020).

22. Hulo, C. et al. ViralZone: a knowledge resource to understand virus diversity. Nucleic Acids Res. 39, D576-D582 (2011).

23. de Wit, E. et al. Prophylactic and therapeutic remdesivir (GS-5734) treatment in the rhesus macaque model of MERS-CoV infection. Proc. Natl Acad. Sci. USA 117, 6771-6776 (2020).

24. Ruch, T. R. \& Machamer, C. E. The coronavirus E protein: assembly and beyond. Viruses 4, 363-382 (2012).

25. Harding, A. T., Heaton, B. E., Dumm, R. E. \& Heaton, N. S. Rationally designed influenza virus vaccines that are antigenically stable during growth in eggs. mBio https://doi.org/10.1128/mBio.00669-17 (2017).

26. Abudayyeh, O. O. et al. A cytosine deaminase for programmable single-base RNA editing. Science 365, 382-386 (2019).

27. Kushawah, G. et al. CRISPR-Cas13d induces efficient mRNA knockdown in animal embryos. Dev. Cell 54, 805-817 (2020).

28. He, B. et al. Modulation of metabolic functions through Cas13d-mediated gene knockdown in liver. Protein Cell https://doi.org/10.1007/s13238-02000700-2 (2020).

29. Zhou, H. et al. Glia-to-neuron conversion by CRISPR-CasRx alleviates symptoms of neurological disease in mice. Cell https://doi.org/10.1016/j. cell.2020.03.024 (2020).

30. Zhou, C. et al. CasRx-mediated RNA targeting prevents choroidal neovascularization in a mouse model of age-related macular degeneration. Natl Sci. Rev. https://doi.org/10.1093/nsr/nwaa033 (2020).

31. Mehta, A. \& Merkel, O. M. Immunogenicity of Cas9 protein. J. Pharm. Sci. 109, 62-67 (2020)

32. East-Seletsky, A. et al. Two distinct RNase activities of CRISPR-C2c2 enable guide-RNA processing and RNA detection. Nature 538, 270-273 (2016).

33. East-Seletsky, A., O’Connell, M. R., Burstein, D., Knott, G. J. \& Doudna, J. A RNA targeting by functionally orthogonal type VI-A CRISPR-Cas enzymes. Mol. Cell 66, 373-383.e373 (2017).

34. Cox, D. B. T. et al. RNA editing with CRISPR-Cas13. Science 358, 1019-1027 (2017)

Publisher's note Springer Nature remains neutral with regard to jurisdictional claims in published maps and institutional affiliations.

(c) The Author(s), under exclusive licence to Springer Nature America, Inc. 2021, corrected publication 2022 


\section{Methods}

Computational identification of the CRISPR-Cas13 systems. Metagenome sequences were downloaded from DOE JGI Integrated Microbial Genomes? A computational pipeline was used to produce an expanded database of class 2 CRISPR-Cas systems from metagenomic sources. CRISPR arrays were identified using Piler- $\mathrm{CR}^{35}$, with all default parameters. Proteins were predicted with Prodiga ${ }^{36}$ in anon mode on all contigs at least $5 \mathrm{~kb}$ in length, and de-duplicated (that is, removing identical protein sequences) to construct a database. Proteins with length between 400 and 900 residues were obtained. RNAfold (http://rna.tbi univie.ac.at/) was used to predict the secondary structure of DR sequences. The NR database was used to remove proteins with significant similarity $\left(E<1 \times 10^{-10}\right)$ to proteins of known function, and Cas proteins in NCBI were used for functional characterization of the candidate Cas 13 proteins. Multiple sequence alignment was then conducted for each candidate Cas effector protein using MAFFT ${ }^{37}$. MEGA ${ }^{38}$ was used to construct the phylogenetic tree. I-TASSER ${ }^{39}$ was used to perform the protein structure prediction.

Natural crRNA target analysis. To search for natural crRNA targets, all crRNA spacer sequences in the CRISPR loci were used as a query to find matched sequences in CRISPSRTarget ${ }^{40}$ in GenBank-phage, RefSeq-plasmid and IMG/VR databases. The 'Mismatch-search.pl' script deposited in our GitHub repository was used to search the natural target in the metagenomic datasets in which the Cas 13 proteins were detected. The natural crRNA targets with no more than five mismatches with each spacer were retained.

Plasmid constructions. Retrieved coding sequences of Cas13X and Cas13Y were human-codon-optimized and synthesized for cloning into SalI/NotI-digested pCX539 backbone with the Gibson assembly method. Predicted DR sequences for each Cas 13 variant were synthesized as oligos for cloning downstream of the human U6 promoter for expression in mammalian cells. A G > A amber mutation was introduced in the mCherry coding sequence to generate mutant fluorescence protein as the RNA base-editing reporter. All primers and Cas 13 sequences used in this study are provided in Supplementary Tables 2 and 11.

Cell culture, transfection and flow cytometry analysis. Mammalian cell lines used in the study were HEK293T and N2A. Media for culturing cells were prepared by supplementing DMEM with $10 \%$ FBS, GlutMAX, sodium pyruvate and penicillin/streptomycin. Transfection of HEK293T and N2A cells was conducted with Lipofectamine 3000 following the manufacturer manual, and cells were analyzed by BD FACSAria II or sorted by MoFlo XDP at $48 \mathrm{~h}$ after transfection. Flow cytometry results were analyzed with FlowJo X (v.10.0.7).

RNA editing and sequencing analysis. To analyze A-to-I or C-to- $\mathrm{U}$ base-editing efficiency of dCas13X, successfully transfected cells were sorted for RNA extraction. RNA was extracted with RNA-easy Isolation Reagent according to the manufacturer protocol. The complementary DNAs were reverse-transcribed from RNAs by HiScript II One Step RT-PCR Kit, and crRNA target sites were amplified from cDNAs with Phanta Max Super-Fidelity DNA Polymerase for Sanger or deep sequencing methods. Deep sequencing libraries were prepared with Nextera XT DNA Library Prep Kit according to the manufacturer manual and sequenced on a HiSeq. Sequencing data were first de-multiplexed by Cutadapt (v.2.8) ${ }^{41}$ based on sample barcodes. The de-multiplexed reads were then processed by CRISPResso2 (ref. ${ }^{42}$ ) for the quantification of A-to-I or C-to-U conversion efficiency at each target site. Sanger sequencing results were analyzed with EditR $^{43}$ to quantify A-to-I or C-to-U conversion efficiency at each target site.

Quantitative PCR with reverse transcription, RNA-seq and analysis. To quantify RNA knockdown efficiency of Cas13 effectors, RNAs were extracted from successfully transfected cells and reverse-transcribed to cDNAs with HiScript II One Step RT-PCR Kit (Vazyme, Biotech). Quantitative PCR (qPCR) was performed with the cDNA for each sample on a Roche 480 II-A, using AceQ Universal SYBR qPCR Master Mix (Vazyme, Biotech). qPCR results were analyzed with the $-\Delta \Delta$ CT method, in which differences between average $C T$ values of target genes and reference gene GAPDH for three biological replicates were used to calculate the relative expression level of the target gene and normalized by that of control groups.

To analyze the functional specificity of Cas 13 effectors, RNAs were extracted with a TRIZOL (Ambion)-based method, fragmented and reverse-transcribed to cDNAs with a HiScript II One Step RT-PCR Kit according to the manufacturer protocol. An RNA-seq library was generated with a TruSeq Stranded Total RNA library preparation kit using the standard protocol. The transcriptome libraries were sequenced using a 150-bp paired-end Illumina Xten platform.

RNA-seq data were analyzed as previously described ${ }^{28}$ and presented as the mean of all repeats. After filtering the low-quality reads with SolexaQA (v.3.1.7.1 $)^{44}$, RNA-seq reads of RNA knockdown experiments were aligned to the hg38 reference genome with Hisat2 (v.2.0.4) ${ }^{45}$. All uniquely mapped reads were processed by HTSeq-count ${ }^{46}$ to generate a read count matrix. DESeq2 (ref. ${ }^{47}$ ) was used to calculate differentially expressed genes. Genes with fold change $>2$ and false discovery rate $(\mathrm{FDR})<0.05$ were treated as differentially expressed genes.
A customized script, HTSeq2FPKM.pl, was used to calculate fragments per kilobase per million mapped fragments (FPKM) values from the read count matrix for plotting visualization.

RNA-seq reads of RNA base-editing experiments were aligned to the hg38 reference genome with Hisat2 (v.2.0.4) ${ }^{45}$. RNA editing sites were calculated using REDItool $s^{48}$ with the following parameters: $-\mathrm{t} 24-\mathrm{e}-\mathrm{d}-1-\mathrm{U}$ [AG or TC or CT or GA] -p -u -m20 - T6-0 -W -v 1 -n 0.0. dbSNP ${ }^{49}$ (v.146) database downloaded from NCBI was used to filter the sites overlapped with common single nucleotide variants (SNVs). The sites with less than ten mutated or nonmutated reads were further filtered.

Prediction of potential off-target sites. Potential off-target sites were identified by a sliding-window method with step size of $1 \mathrm{nt}$. The window-size $(W)$ is the same with target length. The mismatches between potential off-target sites and target site were less than $0.3 \times W$. The 'Mismatch-search.pl' script deposited in our GitHub repository was used to predict the off-target sequences in the hg38 genome and transcriptome. For the genome searching method, if the number of mismatches between any sequence or the reverse complementary sequence of this sequence and the spacer was no more than eight, the sequence was retained as a potential off-target sequence. We further used the 'OffTarget_gene.pl' script in our GitHub repository to identify off-target sequence-associated genes with less than eight mismatches between the forward sequence of genes and the reverse complementary sequence of the spacer. For the transcriptome searching, if a forward sequence of a transcript had no more than eight mismatches with the reverse complementary sequence of the spacer, the transcript was retained. Based on the RNA-seq count matrix generated by 'HTSeq-count', the genes with more than five read counts in at least one sample were labeled as the genes expressed in HEK293 cell line. Finally, the genes obtained by the two methods were combined as suspicious genes with predicted off-target sequences, listed in Supplementary Tables 12-15.

Collateral activity analysis in cell culture. To examine the effect of collateral activity for Cas13X.1/crRNA targeting different genes, we used GFP fluorescence intensity change as the indicator of suspicious collateral activity in a constitutively expressed EGFP-transgenic HEK293T cell line. For endogenous gene knockdown, a plasmid encoding Cas13X.1/crRNA and mCherry was transfected into the cells with Lipofectamine 3000 (L3000008, Thermofisher). For mCherry knockdown, a plasmid encoding Cas13X.1/crRNA and mCherry and a plasmid encoding BFP were cotransfected into the cells. BFP was used for normalizing transfection efficiency difference with Lipofectamine 3000. At $48 \mathrm{~h}$ after transfection, cells were collected and analyzed by BD FACSAria II. Flow cytometry results were analyzed with FlowJo X (v.10.0.7).

PFS analysis. To analyze the PFS requirements for Cas13X.1 activity, target sequences having 16 types of PFS sequence ${ }^{15}$ surrounding the protospacer were designed and cloned upstream of the EGFP gene (designated as PFS-EGFP). Plasmids encoding Cas13X.1/crRNA, mCherry and PFS-EGFP variants were transfected into wild-type HEK293T cells. EGFP knockdown efficiency was analyzed $48 \mathrm{~h}$ after transfection by BD FACSAria II. Fluorescence of mCherry was used as an indicator for successful transfection. Flow cytometry results were analyzed with FlowJo X (v.10.0.7).

Cas13 protein purification. Cas13 protein purification was performed with the protocol previously described ${ }^{16}$. The human-codon-optimized gene for Cas $13 \mathrm{X} / \mathrm{Y} / \mathrm{a} / \mathrm{d}$ was synthesized (Huagene) and cloned into a bacterial expression vector (pC013-Twinstrep-SUMO-huLwCas13a from Dr. Feng Zhang's laboratory, deposited in Addgene as Plasmid no. 90097) after the plasmid digestion by BamHI and NotI with NEBuilder HiFi DNA Assembly Cloning Kit (New England Biolabs). The Cas13X/Y expression constructs were transformed into BL21 (DE3) (TIANGEN) cells. Next, 1 of lysogeny broth (LB) growth medium (tryptone $10.0 \mathrm{~g}$; yeast extract $5.0 \mathrm{~g} ; \mathrm{NaCl} 10.0 \mathrm{~g}$, Sangon Biotech) was inoculated with $10 \mathrm{ml}$ of culture grown for $12 \mathrm{~h}$. Cells were then grown at $37^{\circ} \mathrm{C}$ to a cell density of $0.6 \mathrm{OD}_{600}$, and then SUMO-Cas 13 expression was induced by supplementing with $500 \mathrm{mM}$ isopropylthiogalactoside. The induced cells were grown at $16^{\circ} \mathrm{C}$ for $16-18 \mathrm{~h}$ before collection by centrifuge $(4,000$ r.p.m., $20 \mathrm{~min}$ ). The collected cells were resuspended in Buffer W (Strep-Tactin Purification Buffer Set, IBA) and lysed using an ultrasonic homogenizer (Scientz). Cell debris was removed by centrifugation and the clear lysate was loaded onto a Strep-Tactin Sepharose High Performance Column (StrepTrap HP, GE Healthcare). The nonspecific binding protein and contaminants were flowed through. The target proteins were eluted with elution buffer (Strep-Tactin Purification Buffer Set, IBA). The N-terminal $6 \mathrm{xHis} /$ Twinstrep-SUMO tag was removed by SUMO protease $\left(4^{\circ} \mathrm{C},>20 \mathrm{~h}\right)$. Then, target proteins were subjected to a final polishing step by gel filtration (S200, GE Healthcare). The purity was assessed by SDS-PAGE.

Nuclease assay. A dye-labeled ssRNA reporter assay for Cas13 ribonuclease activity was performed and analyzed as previously described ${ }^{50,51}$. For on-target ribonuclease activity analysis, the assay was performed with $45 \mathrm{nM}$ purified Cas13X/Y/a/d, $22.5 \mathrm{nM}$ crRNA, 5 or $20 \mathrm{nM}$ quenched cy5-labeled target ssRNA reporter (Sangon 
Biotech), $1 \mu \mathrm{l}$ of murine RNase inhibitor (New England Biolabs), $100 \mathrm{ng}$ of background total human RNA (purified from HEK293T cell culture) and varying amounts of input nucleic acid target, unless otherwise indicated, in nuclease assay buffer ( $40 \mathrm{mM}$ Tris- $\mathrm{HCl}$ including $25 \mathrm{mM}$ Tris-HCL, $\mathrm{pH} 7.5$, and $25 \mathrm{mM}$ Tris-HCL, $\mathrm{pH} 7.0,60 \mathrm{mM} \mathrm{NaCl}^{6} \mathrm{mM} \mathrm{MgCl}_{2}, \mathrm{pH} 7.3$ ). For collateral ribonuclease activity analysis, the assay was performed with $45 \mathrm{nM}$ purified Cas $13 \mathrm{X} / \mathrm{Y} / \mathrm{a} / \mathrm{d} ; 22.5 \mathrm{nM}$ crRNA; 0, 5, or 20 target ssRNAs; $125 \mathrm{nM}$ quenched FAM-labeled nontarget ssRNA reporter (Sangon Biotech); $1 \mu \mathrm{l}$ of murine RNase inhibitor; $100 \mathrm{ng}$ of background total human RNA (purified from HEK293T cell culture); and varying amounts of input nucleic acid target, unless otherwise indicated, in nuclease assay buffer. Reactions were allowed to proceed for $1-3 \mathrm{~h}$ at $37^{\circ} \mathrm{C}$ on a fluorescence plate reader (Analytik Jena) with fluorescence kinetics measured every $5 \mathrm{~min}$.

Antiviral experimental and analysis method. MDCK cells were seeded onto 96-well plates and incubated with DMEM (Gibco) supplemented with $10 \%$ fetal bovine serum (FBS; Gibco) and 1\% penicillin/streptomycin. The cells were further infected with influenza A virus H1N1 (A/Puerto Rico/8/1934) ${ }^{25}$ at 100 times doses of median tissue culture infective dose $\left(\mathrm{TCID}_{50}\right)$. At $1 \mathrm{~h}$ post-infection, the medium was replaced with DMEM containing $0.1 \%$ BSA and $1 \mu \mathrm{g} \mathrm{ml}^{-1}$ of TPCK-trypsin. At $48 \mathrm{~h}$ post-infection, supernatant and cell lysate were collected for measuring viral titers. Total RNA was extracted from supernatants of virus-infected MDCK cells, and quantitative PCR with reverse transcription (RT-qPCR) was performed using influenza virus-specific primers for determination of relative levels of viral loads. All primers used in this study are provided in Supplementary Table 11.

Statistical analysis. All values are shown as mean \pm s.e.m. Unpaired Student's $t$-test (two-tailed) was used for comparisons and $P<0.05$ was considered to be statistically significant. Details of statistical values are provided in Source Data Figs. 1-4 and Extended Data Figs. 1-10. The experiments were not randomized and the investigators were not blinded to allocation during experiments and outcome assessment.

Material availability. All materials are available upon reasonable request.

Reporting Summary. Further information on research design is available in the Nature Research Reporting Summary linked to this article.

\section{Data availability}

All the sequencing data have been deposited in the NCBI SRA under project accession number PRJNA680488. Meta-information on all raw NGS datasets is provided in Supplementary Table 16. Source data are provided with this paper.

\section{Code availability}

Bioinformatics codes were deposited in the GitHub repository (https://github.com/ yszhou2016/Cas13).

\section{References}

35. Edgar, R. C. PILER-CR: fast and accurate identification of CRISPR repeats. BMC Bioinformatics 8, 18 (2007).

36. Hyatt, D. et al. Prodigal: prokaryotic gene recognition and translation initiation site identification. BMC Bioinformatics 11, 119 (2010).

37. Katoh, K. \& Standley, D. M. MAFFT multiple sequence alignment software version 7: improvements in performance and usability. Mol. Biol. Evolution 30, 772-780 (2013).

38. Kumar, S., Stecher, G. \& Tamura, K. MEGA7: Molecular Evolutionary Genetics Analysis version 7.0 for bigger datasets. Mol. Biol. Evolution 33, 1870-1874 (2016).

39. Zhang, Y. I-TASSER server for protein 3D structure prediction. BMC Bioinformatics 9, 40 (2008).

40. Biswas, A., Gagnon, J. N., Brouns, S. J., Fineran, P. C. \& Brown, C. M. CRISPRTarget: bioinformatic prediction and analysis of crRNA targets. RNA Biol. 10, 817-827 (2013).
41. Martin, M. Cutadapt removes adapter sequences from high-throughput sequencing reads. EMBnet J. https://doi.org/10.14806/ej.17.1.200 (2011)

42. Clement, K. et al. CRISPResso2 provides accurate and rapid genome editing sequence analysis. Nat. Biotechnol. 37, 224-226 (2019).

43. Kluesner, M. G. et al. EditR: a method to quantify base editing from Sanger sequencing. CRISPR J. 1, 239-250 (2018).

44. Cox, M. P., Peterson, D. A. \& Biggs, P. J. SolexaQA: at-a-glance quality assessment of Illumina second-generation sequencing data. BMC Bioinformatics 11, 485 (2010).

45. Kim, D., Langmead, B. \& Salzberg, S. L. HISAT: a fast spliced aligner with low memory requirements. Nat. Methods 12, 357-360 (2015).

46. Anders, S., Pyl, P. T. \& Huber, W. HTSeq-a Python framework to work with high-throughput sequencing data. Bioinformatics 31, 166-169 (2015).

47. Love, M. I., Huber, W. \& Anders, S. Moderated estimation of fold change and dispersion for RNA-seq data with DESeq2. Genome Biol. 15, 550 (2014).

48. Picardi, E. \& Pesole, G. REDItools: high-throughput RNA editing detection made easy. Bioinformatics 29, 1813-1814 (2013).

49. Smigielski, E. M., Sirotkin, K., Ward, M. \& Sherry, S. T. dbSNP: a database of single nucleotide polymorphisms. Nucleic Acids Res. 28, 352-355 (2000).

50. Gootenberg, J. S. et al. Nucleic acid detection with CRISPR-Cas13a/C2c2. Science 356, 438-442 (2017).

51. Tambe, A., East-Seletsky, A., Knott, G. J., Doudna, J. A. \& O’Connell, M. R. RNA binding and HEPN-nuclease activation are decoupled in CRISPR-Cas13a. Cell Rep. 24, 1025-1036 (2018).

\section{Acknowledgements}

We thank M. Poo for discussions and comments on this manuscript; and the FACS facility, H. Wu and L. Quan at ION. This work was supported by the Basic Frontier Scientific Research Program of Chinese Academy of Sciences From 0 to 1 original innovation project (grant no. ZDBS-LY-SM001), the R\&D Program of China (grant nos. 2017YFC1001300 and 2018YFC2000100), the CAS Strategic Priority Research Program (grant no. XDB32060000), the National Natural Science Foundation of China (grant nos. 31871502, 31925016, 91957122, 31901047, 82021001), the Shanghai Municipal Science and Technology Major Project (grant no. 2018SHZDZX05), the Shanghai City Committee of Science and Technology Project (grant nos. 18411953700, 18JC1410100 19XD1424400, 19YF1455100) and the International Partnership Program of Chinese Academy of Sciences (grant no. 153D31KYSB20170059).

\section{Author contributions}

C.X., Y.Z. and H.Y. conceived the project. C.X., Y.Z., Q.X., B.H. and G.G. designed and conducted experiments. Y.Z. and J.L. performed bioinformatics analysis. Z.W. and B.C. assisted with plasmids construction and RNA analysis. T.Y. assisted with cell experiments. X.W., D.Z. and X.H. assisted with virus experiments. H.Y. designed experiments and supervised the whole project. C.X., Y.Z. and H.Y. wrote the paper.

\section{Competing interests}

H.Y. is a founder of Hui-Gene Therapeutics. H.Y., C.X., Y.Z., and Q.X. are co-inventors on US patent application 16/864,982 relating to the Cas proteins described in this manuscript. The remaining authors declare no competing interests.

\section{Additional information}

Extended data is available for this paper at https://doi.org/10.1038/s41592-021-01124-4. Supplementary information The online version contains supplementary material available at https://doi.org/10.1038/s41592-021-01124-4.

Correspondence and requests for materials should be addressed to Y.Z., J.L. or H.Y. Peer review information Nature Methods thanks the anonymous reviewers for their contribution to the peer review of this work. Lei Tang was the primary editor on this article and managed its editorial process and peer review in collaboration with the rest of the editorial team.

Reprints and permissions information is available at www.nature.com/reprints. 


\section{a}

\section{Our pipeline}

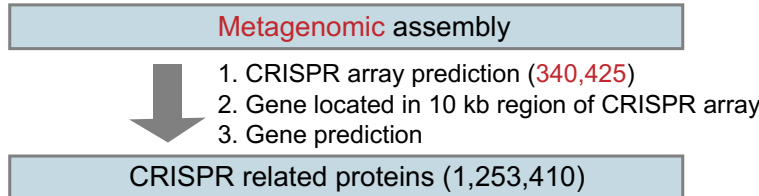

1. With length ranges from 400 to 900 amino acids 2. Within 10 protein-coding genes of repeat

\section{CRISPR related proteins $2(250,901)$}

Two RxxxxH motifs in the nearby region of $\mathrm{N}$ terminus and $\mathrm{C}$ terminus

\section{RxxxxH proteins $(24,959)$}

Two RNxxxH / RHxxxH / RQxxxH motifs in the nearby region of $\mathrm{N}$ terminus and $\mathrm{C}$ terminus

Candidate Cas 13 proteins (64)

1. Direct repeat with steem loop structure 2. Remove proteins with significant similarity $($ E-value $<1 e-10)$ to proteins of known function in NR database

Novel Cas 13 proteins (6)

BLASTP novel Cas 13 proteins against RxxxxH proteins

Novel Cas 13 proteins families (2 families, 7 proteins)

b

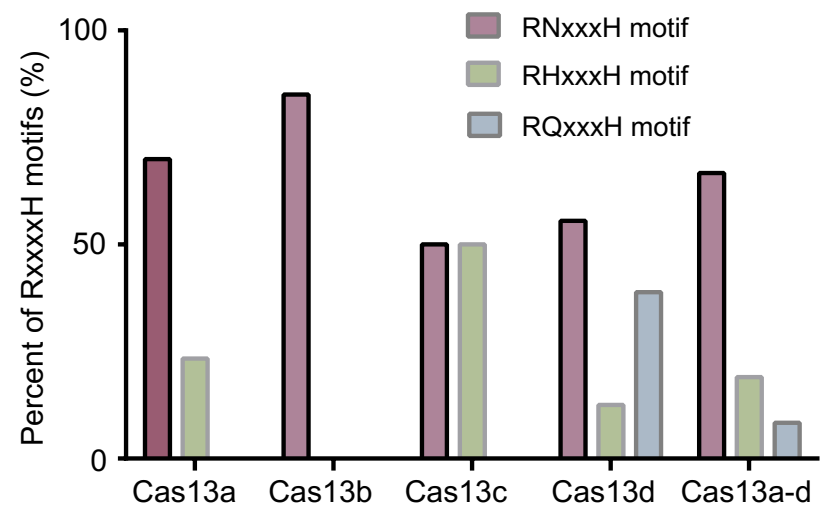

d

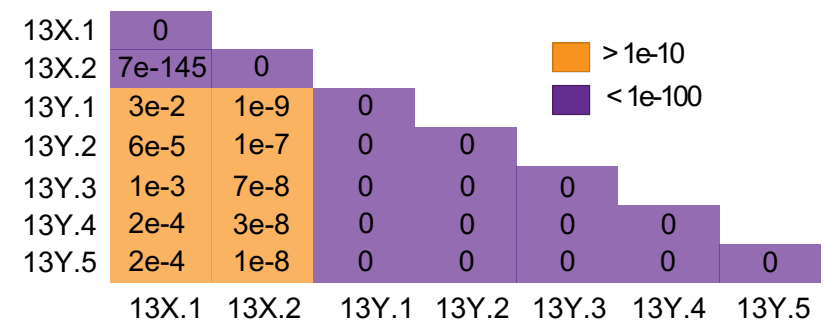

Classical pipeline (Cas13d)

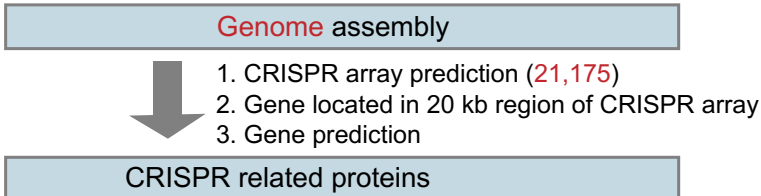

1. With length $>750$ amino acids

2 . Within 5 protein-coding genes of repeat

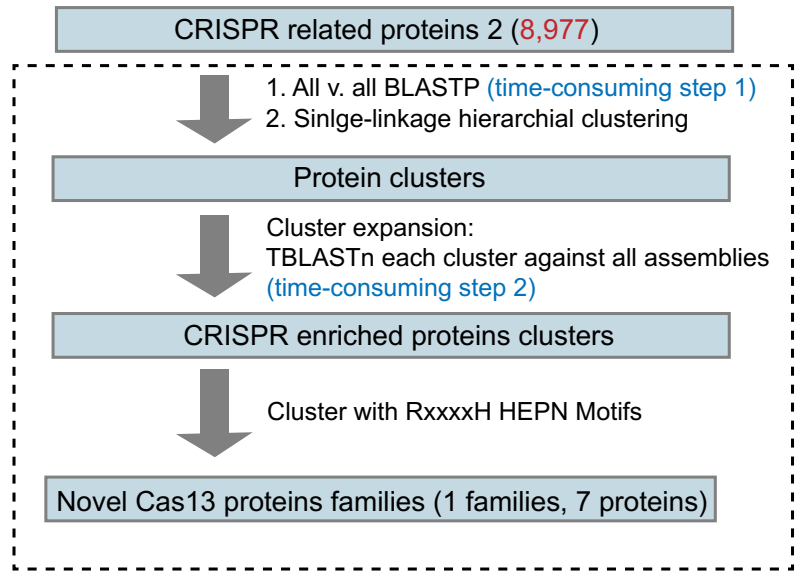

C

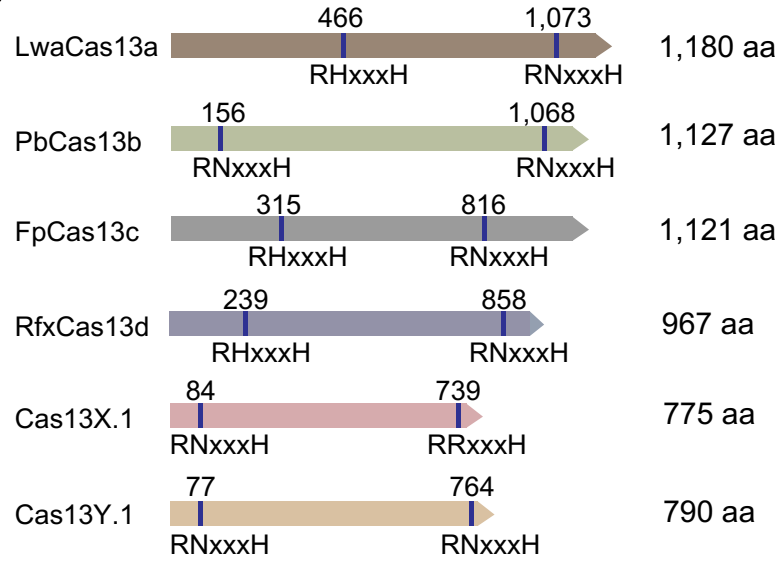

e

\section{\begin{tabular}{lll}
\multicolumn{3}{c}{ HEPN-1 } \\
\hline$* * *$ & $* * * * * *$ & $*$
\end{tabular}}

Cas13X.1 QAANAKAEALRNYFSHYRHSPGCLTFT Cas13X.2 TTANRKLKDIRNYFSHHYHKNECLYFK

Cas13Y 1DCHLKL Cas13Y.2DCLLFKLEELRNFYSHYMHTDMKELS Cas13Y.3 DCLLLKLRELRNFYSHYYTDDVKILS Cas13Y.4 EAILLKLVELRNFYSHMHNDTVKILS Cas13Y.5 EALLLKLVELRNFYSHYMHKDDVKTLS LwaCas13a ANIDEAISSIRHGIVHFNLELEGKDIF PspCas13b KRAFGVLKMYRDLTNHYKTYEEKLNDG RfxCas13d YDILALLSGLRHWWHNNEEESRISRT

\begin{tabular}{c} 
HEPN-2 \\
\hline$* \quad * * * \quad * * *$ \\
EAEKTAVNKVRRAFFHHHLKFVIDEFG \\
KDEQDDLKKVRNSLLHYKLIFEKEHLK \\
$* \quad * * * * * * * * \quad * * *$ \\
KDRLTKLKDARNKALHGEILTGTSFDE \\
RNKLTELNNARNAALHGEIPSETSFRE \\
KDKLTKLKDARNAALHGEIPAETSFRE \\
TEKLSKLKHARNKALHGEIPDGTSFEK \\
ENKIIKLKDARNAALHGKIPEDTSFDE \\
LKQEKKDLYIRNYIAHFNYIPHAEISL \\
KEQSDILRKIRNAFDHNNYPDKGWEI \\
RIDNKTCTLFRNKAVHLEVARYHAYI
\end{tabular}

Extended Data Fig. 1 | See next page for caption. 
Extended Data Fig. 1 | Computational pipeline for the identification of new CRISPR RNA-Targeting System Cas13 and protein schematics for Cas13 effectors. a, Schematic describing a fast and classical computational pipeline for CRISPR system identification. In fast pipeline, a minimal definition for a putative class 2 CRISPR locus was used, requiring only a CRISPR repeat array and a nearby protein of 400 to 900 aa in length. $\mathbf{b}$, Distribution of $\mathrm{RN} x x \mathrm{xH}, \mathrm{RH} x \mathrm{x} x \mathrm{H}, \mathrm{RQ} x \mathrm{xxH}$ motifs among Cas13 proteins. All values are presented as percentage of motif occurrence $(\mathrm{n}=180)$. c, Schematics describing protein-size and HEPN motif position of previously identified Cas13a/b/c/d and Cas13X/f proteins used in this study. The RxxxxH HEPN motif is highlighted. d, Similarity analysis between type $\mathrm{E}$ and $\mathrm{F}$ effectors by position-specific iterated BLAST. E value of $>1$ - 10 defined as cutoff for non-significant similarity. e, HEPN domain alignment between type VI-X/Y and previously reported Cas 13 effectors. Conserved residuals for Cas $13 \mathrm{X}$ and Cas $13 \mathrm{Y}$ were highlighted in pink and previously reported Cas13 in green. 


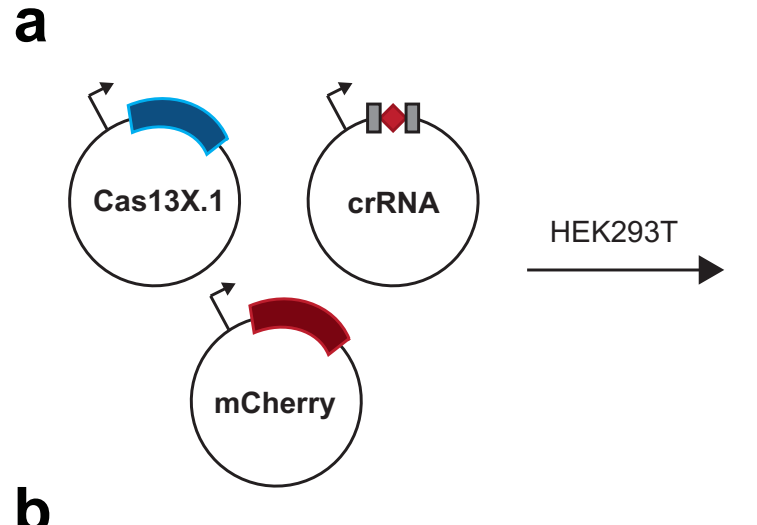

b

Cas13X.1

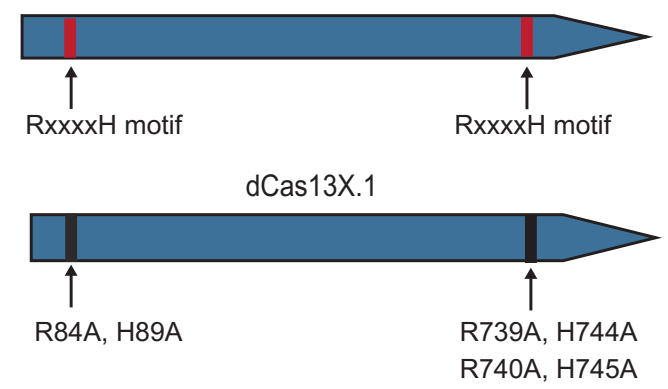

d

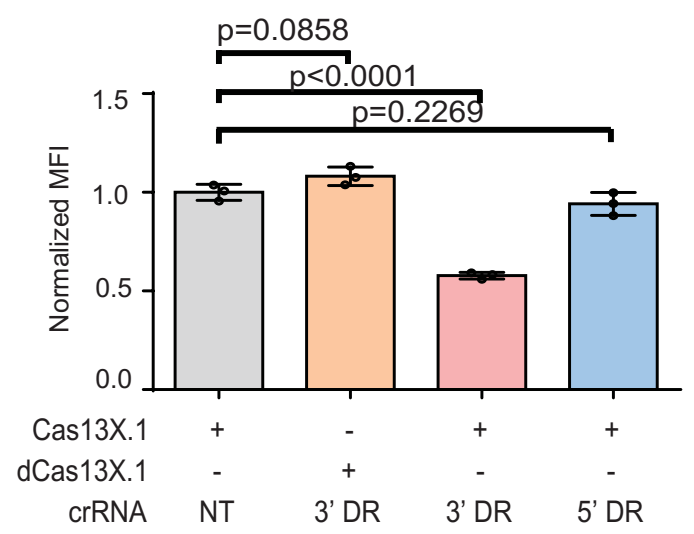

f

Target
AAAATTATTCCTTCCATCATCCCGACAGCAGTGGAAAA
AAAATTATTCCTTCCATCATCCCGACAGCAGTGGTTTT
AAAATTATTCCTTCCATCATCCCGACAGCAGTGGCCCC
AAAATTATTCCTTCCATCATCCCGACAGCAGTGGGGG
TTTTTTATTCCTTCCATCATCCCGACAGCAGTGGAAAA
TTTTTTATTCCTTCCATCATCCCGACAGCAGTGGTTTT
TTTTTTATTCCTTCCATCATCCCGACAGCAGTGGCCCC
TTTTTTATTCCTTCCATCATCCCGACAGCAGTGGGGG
CCCCTTATTCCTTCCATCATCCCGACAGCAGTGGAAAA
CCCCTTATTCCTTCCATCATCCCGACAGCAGTGGTTTT
CCCCTTATTCCTTCCATCATCCCGACAGCAGTGGCCCC
CCCCTTATTCCTTCCATCATCCCGACAGCAGTGGGGGG
GGGGTTATTCCTTCCATCATCCCGACAGCAGTGGAAAA
GGGGTTATTCCTTCCATCATCCCGACAGCAGTGGTTTT
GGGGTTATTCCTTCCATCATCCCGACAGCAGTGGCCCC
GGGGTTATTCCTTCCATCATCCCGACAGCAGTGGGGGG

Normalized MFI

$0.0 \quad 0.5 \quad 1.0 \quad 1.5$

3

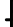

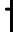

3
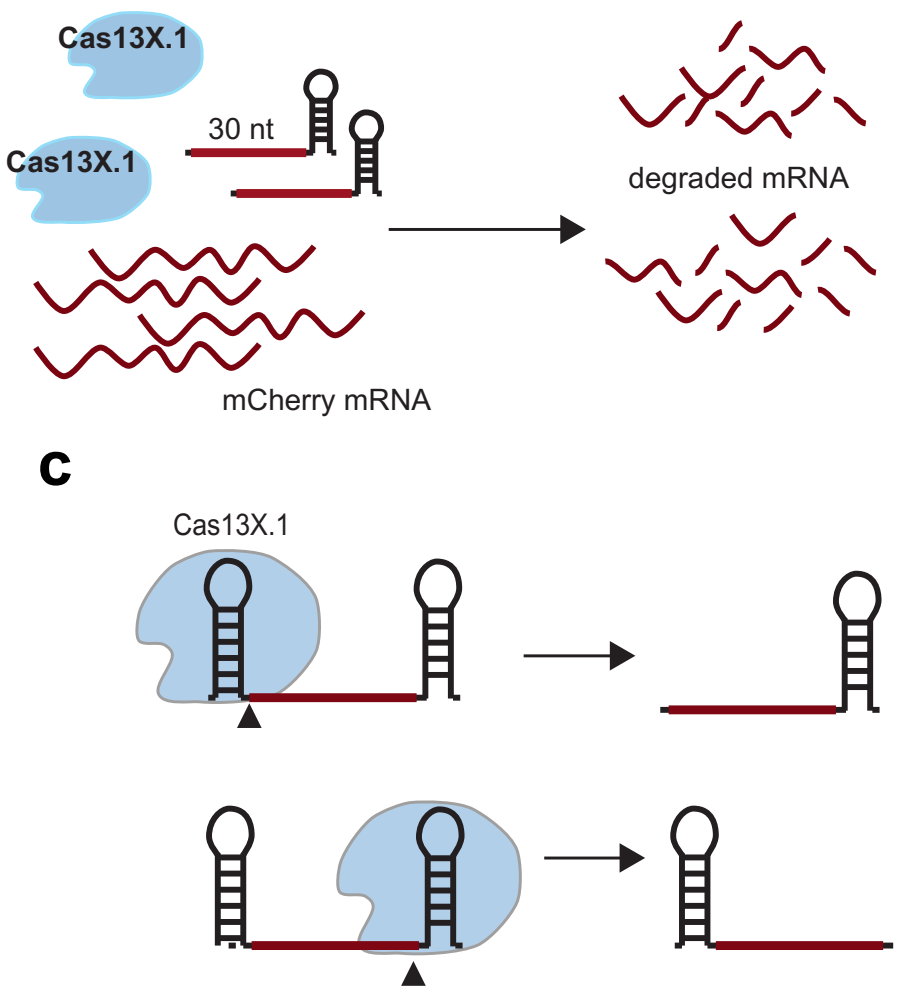

e
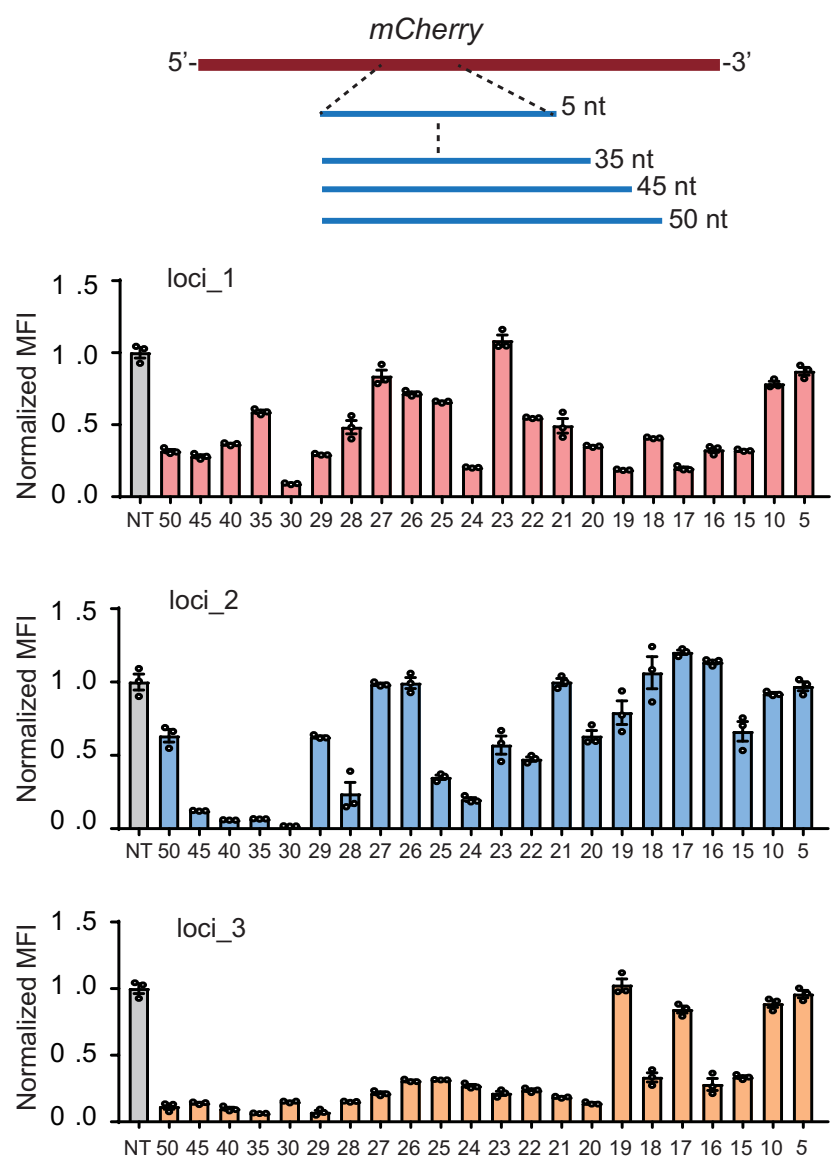
Extended Data Fig. 2 | Pre-crRNA processing mechanism of Cas13X.1 and effect of crRNA length and PFS on Cas13X.1 activity. a, Procedure diagram of mCherry reporter inhibition assay for testing Cas13 activity in HEK293T. b. Schematics describing HEPN-active and -inactive Cas13X.1. The wild-type and inactivated mutant RxxxxH HEPN motifs are highlighted. $\mathbf{c}$, Schematic diagram of potential mechanisms for pre-crRNA to mature crRNA processing by Cas13X.1. d, Reporter inhibition assay revealed ribonuclease-activity dependence on HEPN domains and crRNAs with 3'DR. e, Top, Schematic diagram showing the mCherry specific crRNAs with different spacer lengths. Bottom, Changes of mCherry fluorescence intensity for mCherry specific crRNAs of different lengths relative to non-targeting (NT) crRNA, as measured by flow cytometry. f, PFS analysis with crRNA targeting sequences flanked by different PFS. All values are presented as mean \pm s.e.m $(n=3)$. P values are by two-sided unpaired t-test. 
a
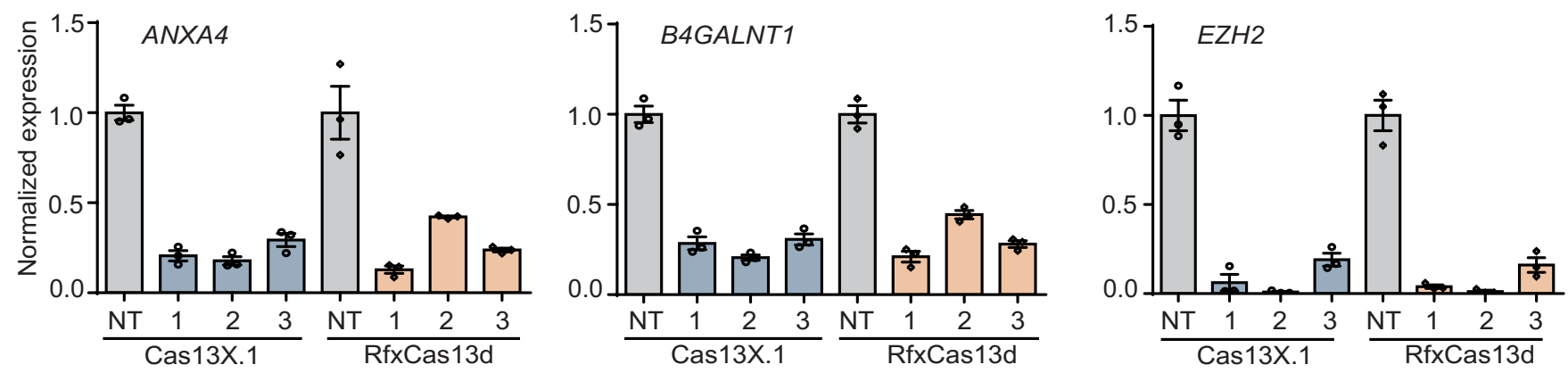

b
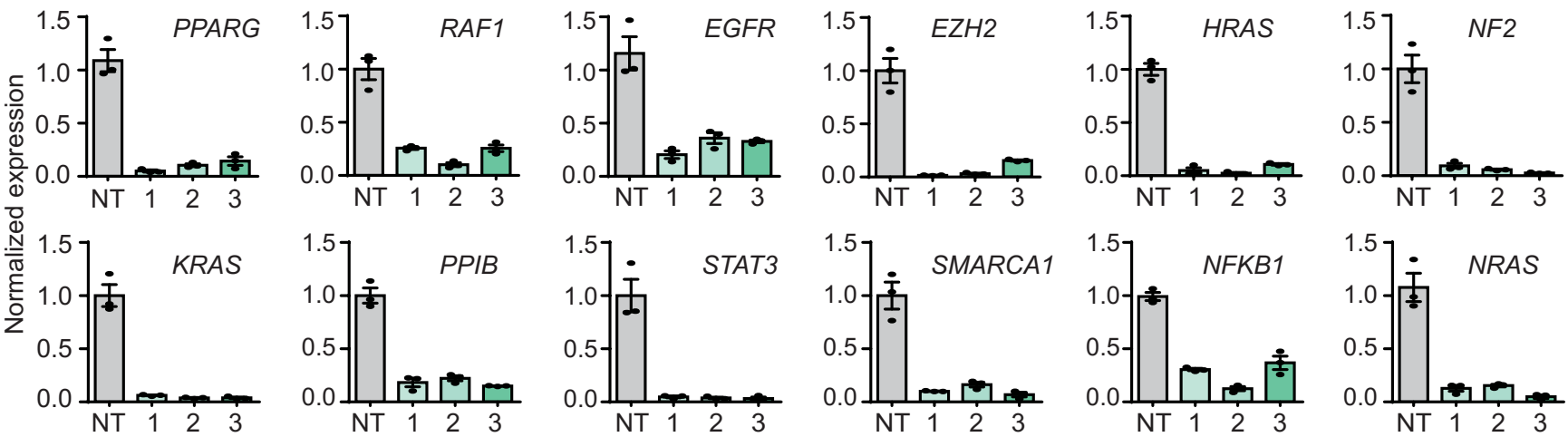

C
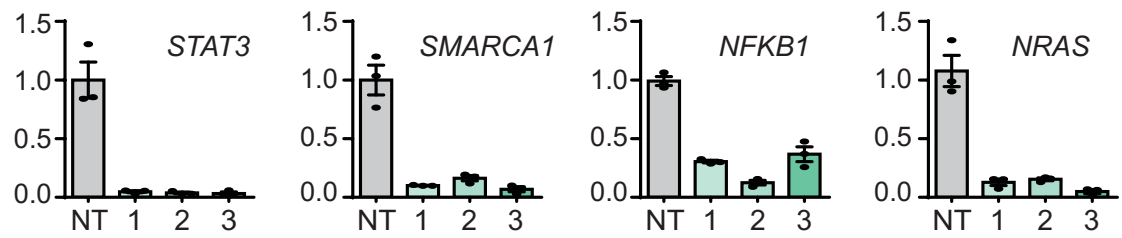

mCherry

d

endogenous gene
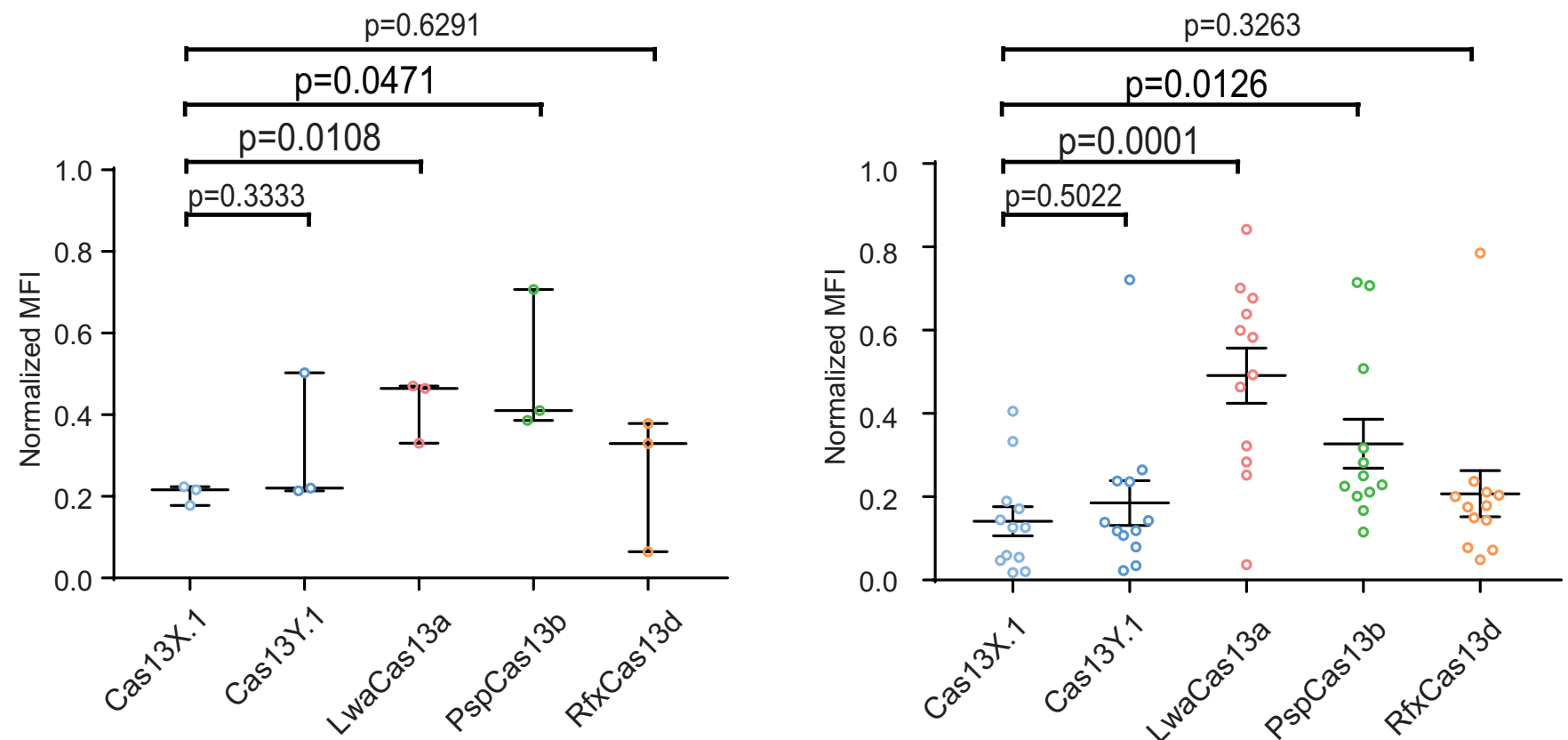

Extended Data Fig. 3 | Comparison of knockdown activity between previously reported Cas13 and Cas13X.1/Cas13Y.1. a, Relative target RNA knockdown by individual position-matched Cas13X.1 and RfXCasRx crRNAs. b, Cas13X.1 targeting efficiency of 12 endogenous transcripts, each with 3 guides and a non-targeting (NT) crRNA in HEK293T cells. c, Comparison of mCherry knockdown activity among Cas13X.1, Cas13Y.1, LwaCas13a, PspCas13b and RfXCas13d ( $n=3$ for each protein). d, Comparison of endogenous genes knockdown activity among Cas13X.1, Cas13Y.1, LwaCas13a, PspCas13b and $\operatorname{Rfx} \operatorname{Cas} 13 d\left(n=12\right.$ for each protein). ${ }^{\star} P<0.05,{ }^{\star \star \star} P<0.001$, two-sided unpaired t-test. All values are presented as mean \pm s.e.m $(n=3)$. $P$ values are by two-sided unpaired t-test. 
Cas13X.1_B4GALNT1

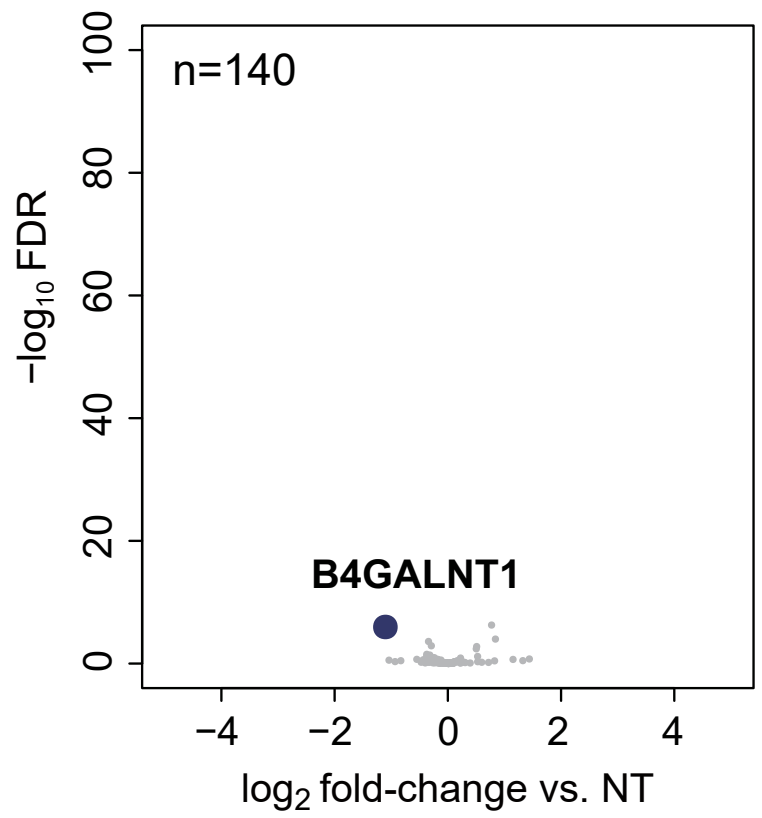

Cas13X.1_EZH2

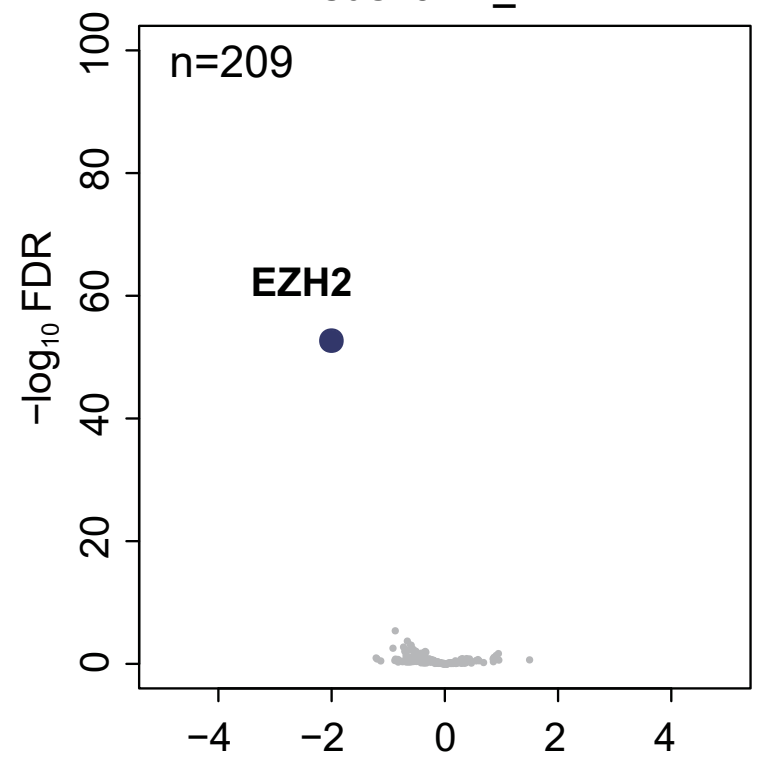

$\log _{2}$ fold-change vs. NT
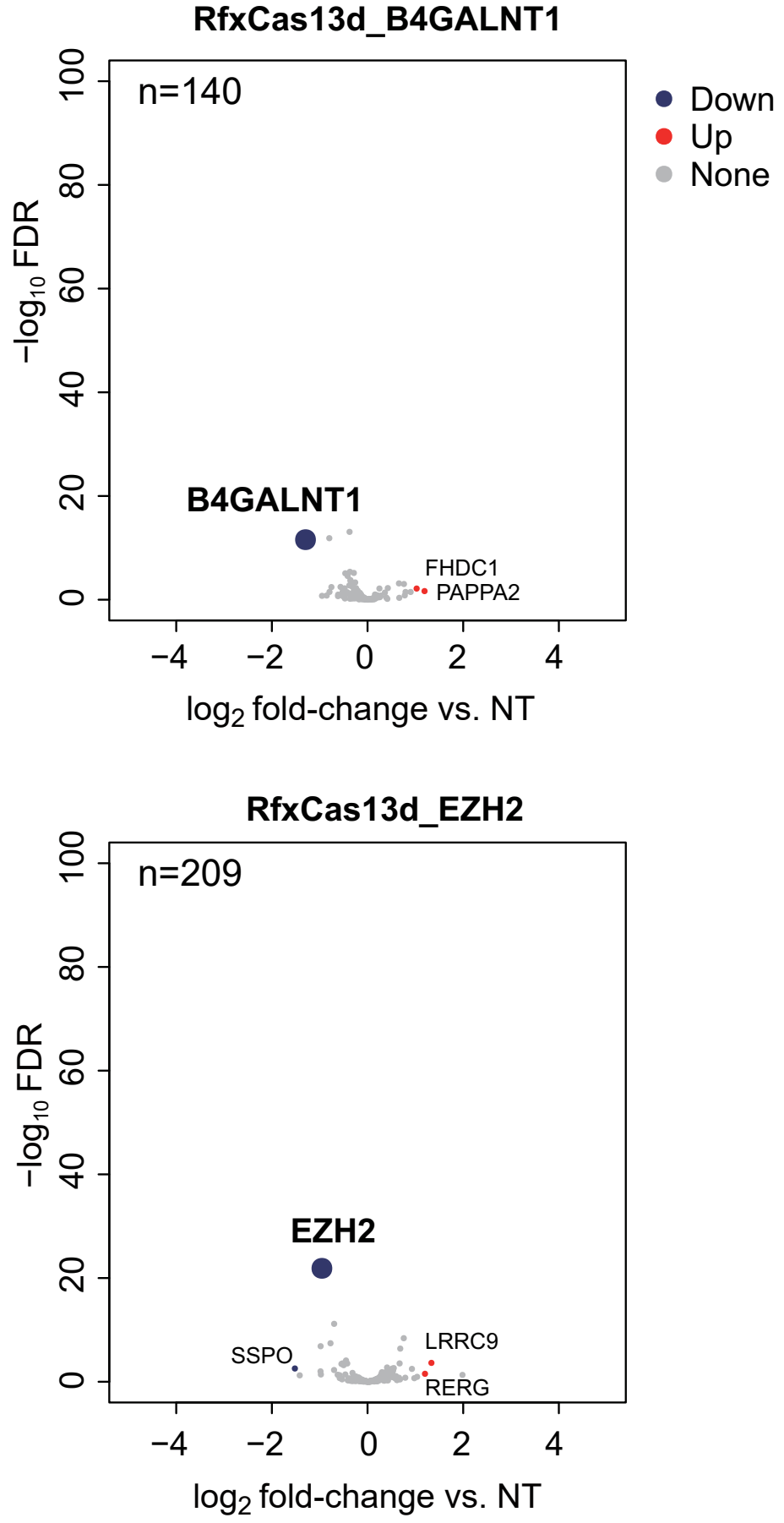

Extended Data Fig. 4 | Genome-wide search for similar sequences with crRNA target from both B4GALNT1 and EZH2 gene predicted several potential off-target genes and volcano plot showed their differential expression significance. Red and blue dot indicate significantly upregulated and downregulated off-target genes. Grey dot depicts non-significantly regulated off-target genes. Large blue dot indicates target gene, B4GALNT1 and EZH2. 
a

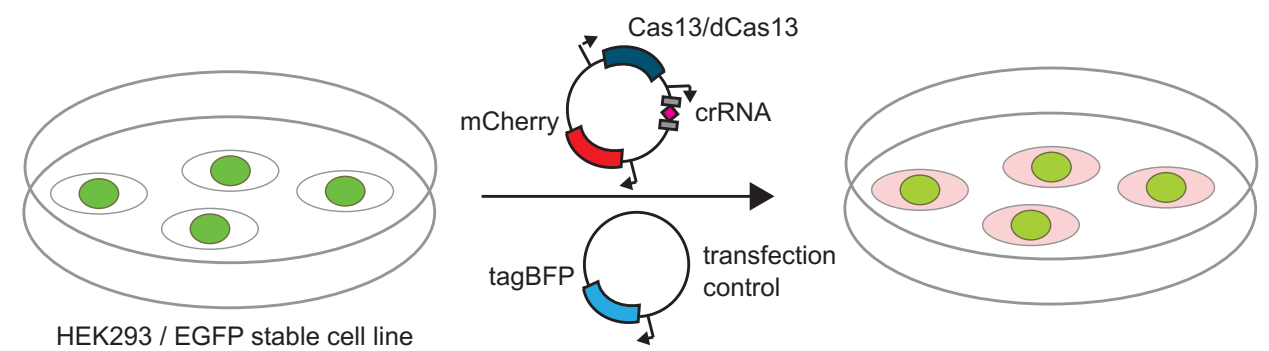

b
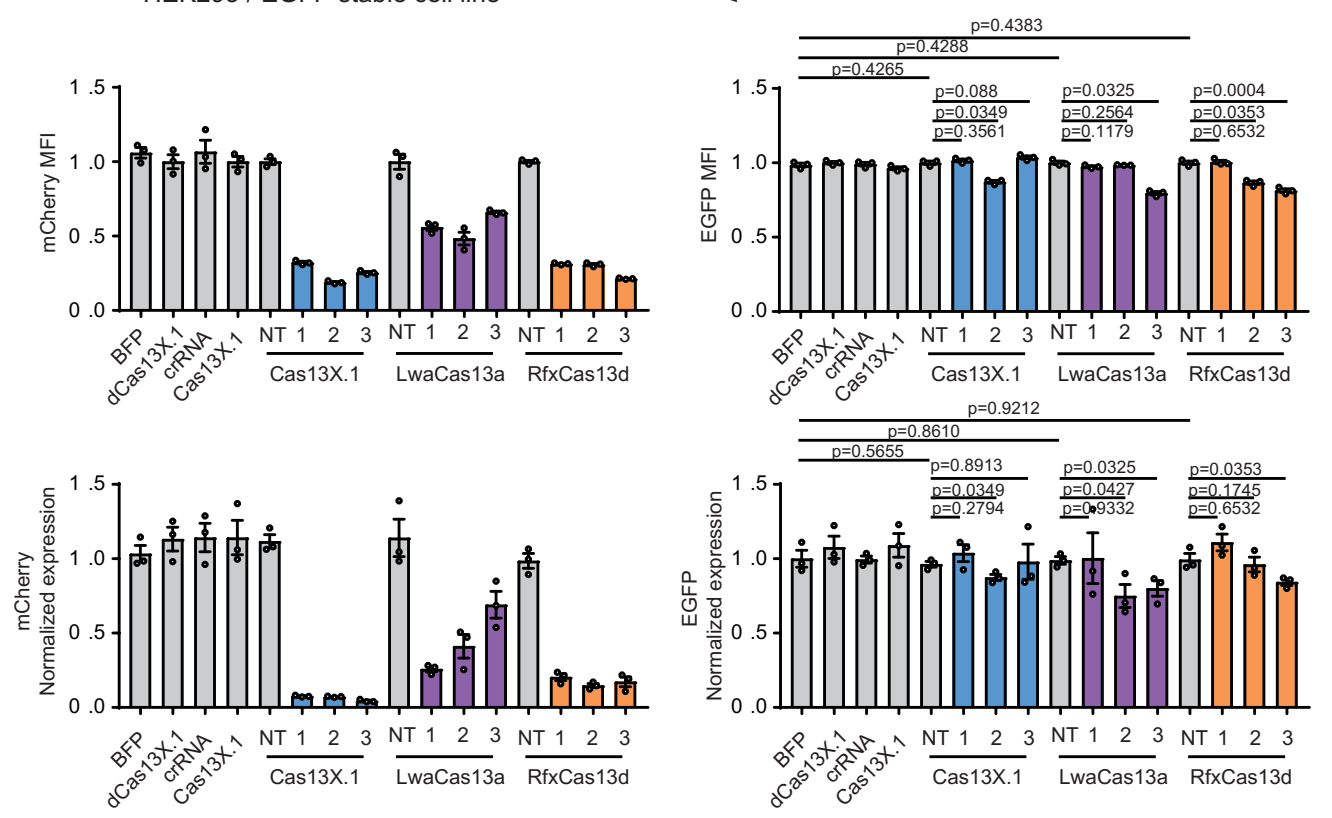

C
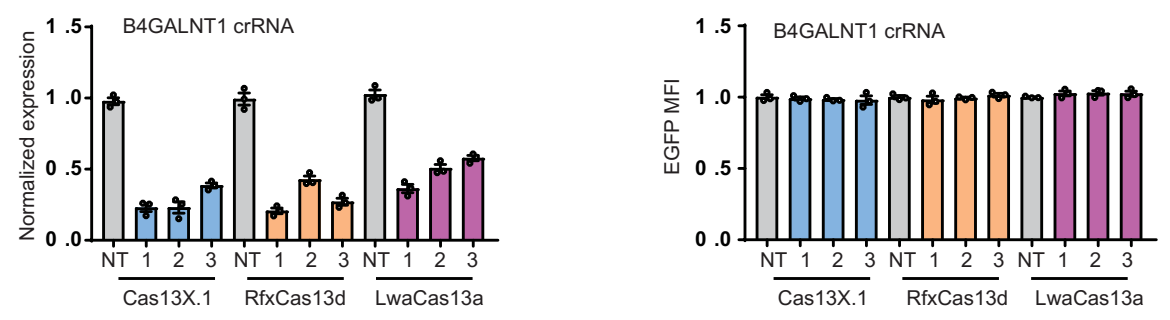

d
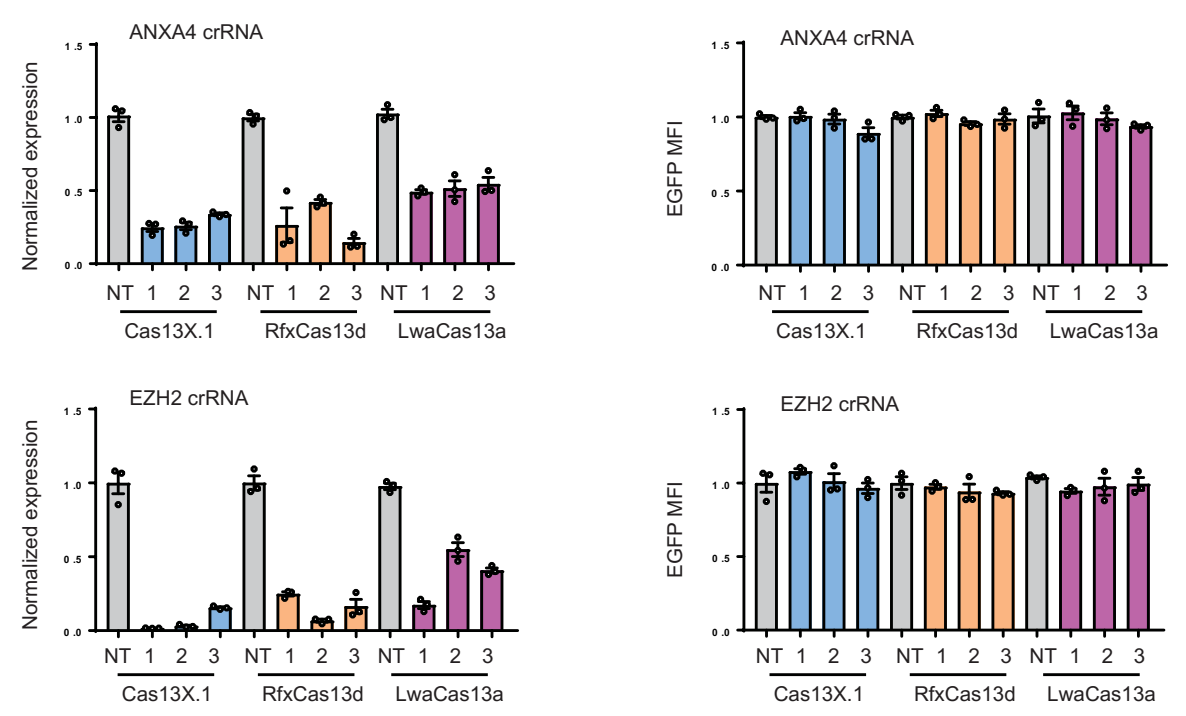

Extended Data Fig. 5 | See next page for caption. 
Extended Data Fig. 5 | Collateral activity comparison between Cas13X.1, RfxCas13d and LwaCas13a in HEK293T cells. a, Schematics describing collateral activity detection system based on EGFP-transgenic HEK293T cells. b-e, Target knockdown and collateral activity for Cas13X.1, RfxCas13d and LwaCas13a with mCherry, endogenous ANXA4, B4GALNT1 and EZH2-targeting crRNAs. All values are presented as mean \pm s.e.m $(n=3)$. P values are by two-sided unpaired t-test. 
a

On-target ribonuclease activity

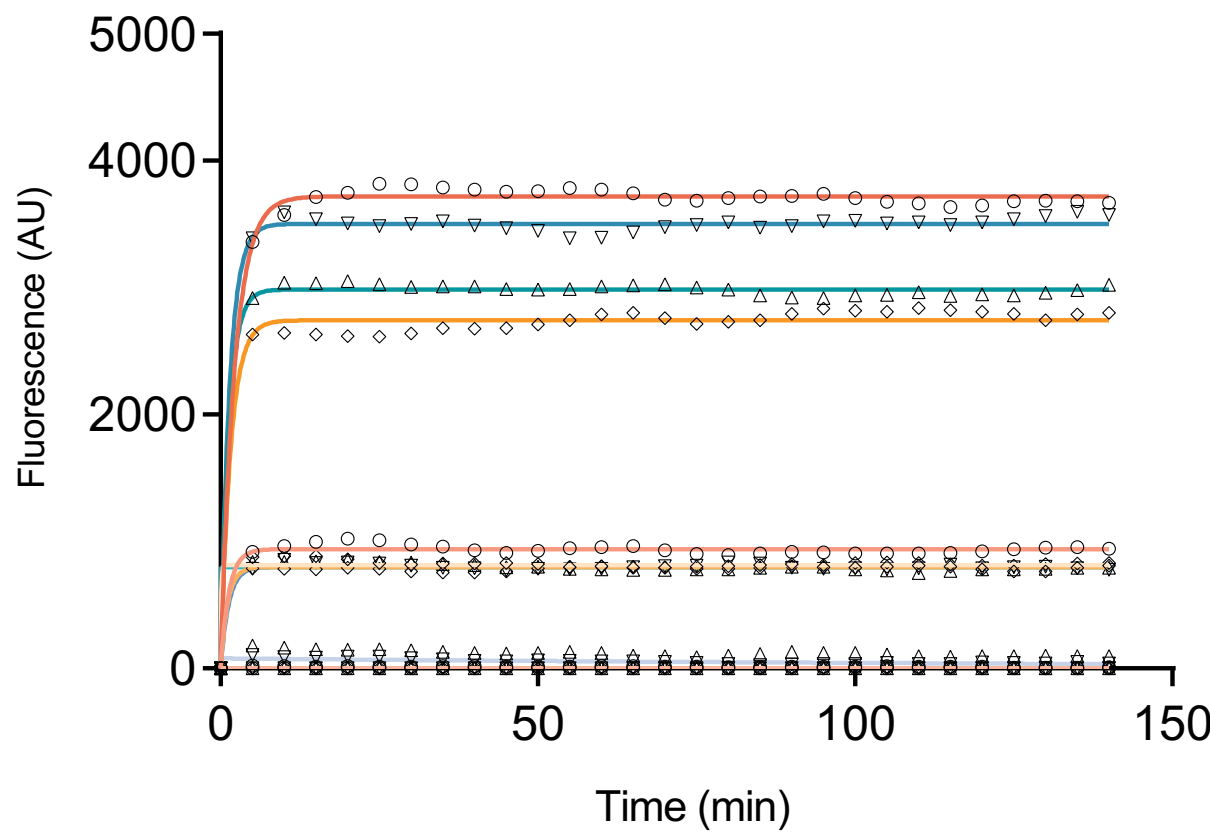

Collateral ribonuclease activity

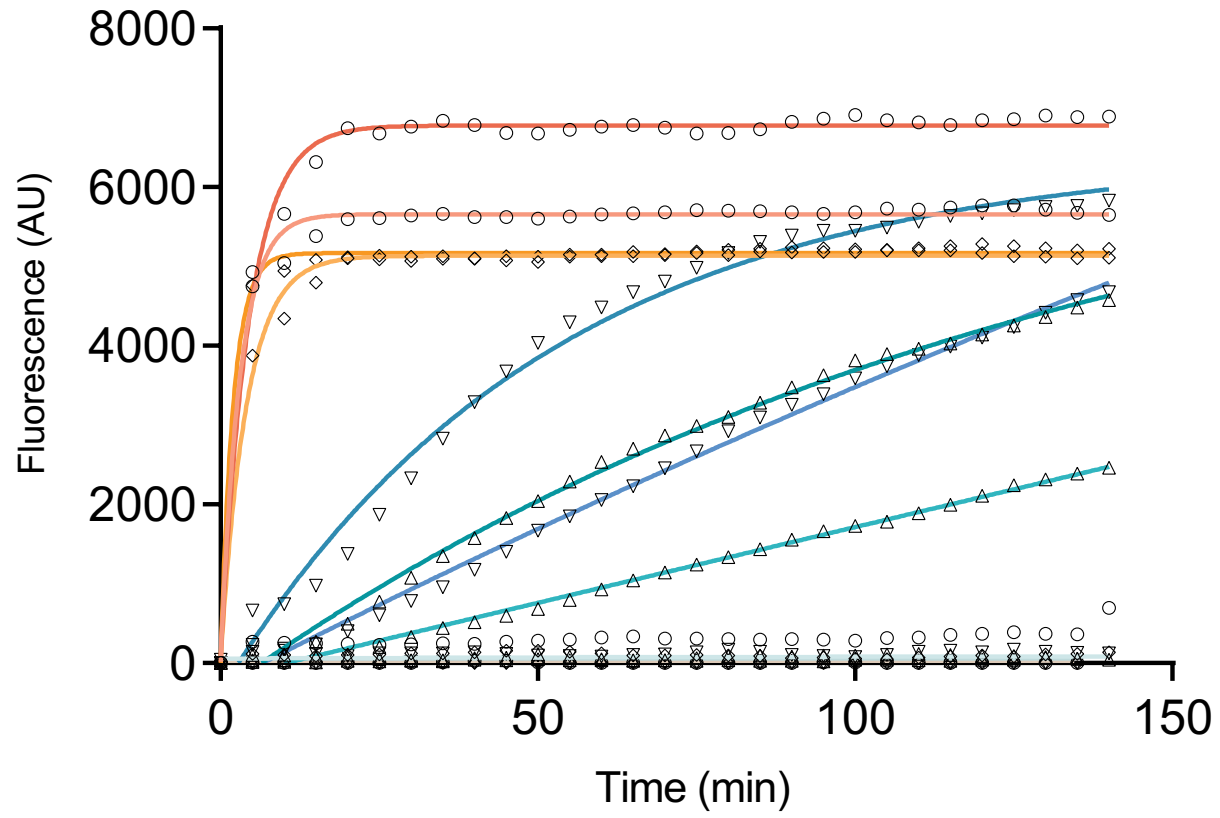

- LwaCas13a

_- OnM Cy5 Target ssRNA

- 5 nM Cy5 Target ssRNA

- 20 nM Cy5 Target ssRNA crRNA

- RfxCas13d

$\therefore$ 0nM Cy5 Target ssRNA

$\multimap$ 5nM Cy5 Target ssRNA

$\therefore 20 n M$ Cy5 Target ssRNA

$\triangle$ Cas13X.1

$\triangle$ 0nM Cy5 Target ssRNA

$\triangle 5 \mathrm{nM}$ Cy5 Target ssRNA

\begin{tabular}{r|l}
$\triangle$ & + \\
& \\
& crRNA
\end{tabular}

$\rightarrow$ Cas $13 Y .1$

$\neg-$ OnM Cy5 Target ssRNA

$\rightarrow$ 5nM Cy5 Target ssRNA crRNA

$\rightarrow$ 20nM Cy5 Target ssRNA $\mid$ CrRNA

FAM non-target ssRNA

- LwaCas13a

- Onm Target ssRNA

- $5 \mathrm{~nm}$ Target ssRNA

$-20 \mathrm{~nm}$ Target ssRNA crRNA

- RfxCas13d

$\therefore$ Onm Target ssRNA

$\leadsto 5 \mathrm{~nm}$ Target ssRNA

$-20 n m$ Target ssRNA $\mid$ crRNA

$\triangle$ Cas13X.1

$\triangle$ Onm Target ssRNA

$\triangle 5 \mathrm{~nm}$ Target ssRNA

$\triangle$ 20nm Target ssRNA $\mid \begin{aligned} & + \\ & \text { crRNA }\end{aligned}$

$\rightarrow$ Cas $13 Y .1$

\begin{tabular}{l|l}
$\nabla-0 \mathrm{~nm}$ Target ssRNA & Cas13Y.1 \\
$\nabla-5 \mathrm{~nm}$ Target ssRNA & + \\
& crRNA \\
$\nabla \quad 20 \mathrm{~nm}$ Target ssRNA &
\end{tabular}

Extended Data Fig. 6 | See next page for caption. 
Extended Data Fig. 6 | Biochemical characterization of on-target and collateral ribonuclease activity. a, On-target ribonuclease activity comparison among LwaCas13a, RfxCas13d, Cas13X.1 and Cas13Y.1. b, Collateral ribonuclease activity comparison among LwaCas13a, RfxCas13d, Cas13X.1 and Cas13Y.1. $\mathrm{AU}$, arbitrary unit. All Values shown are mean $(n=3)$. 
a

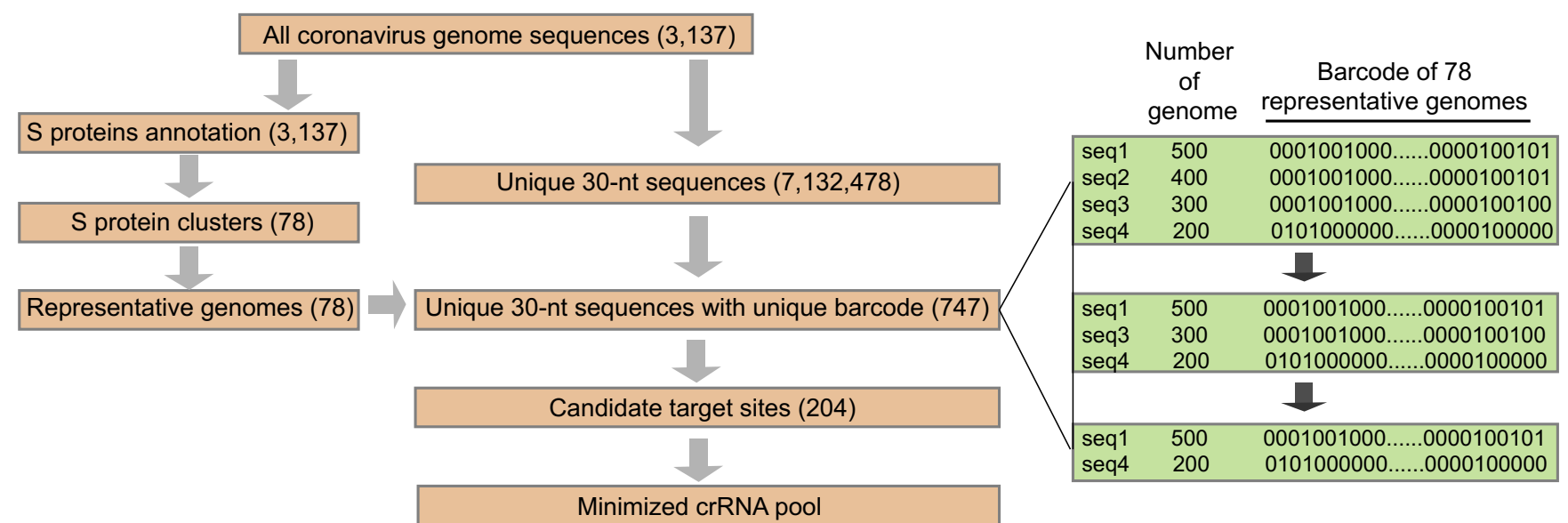

b

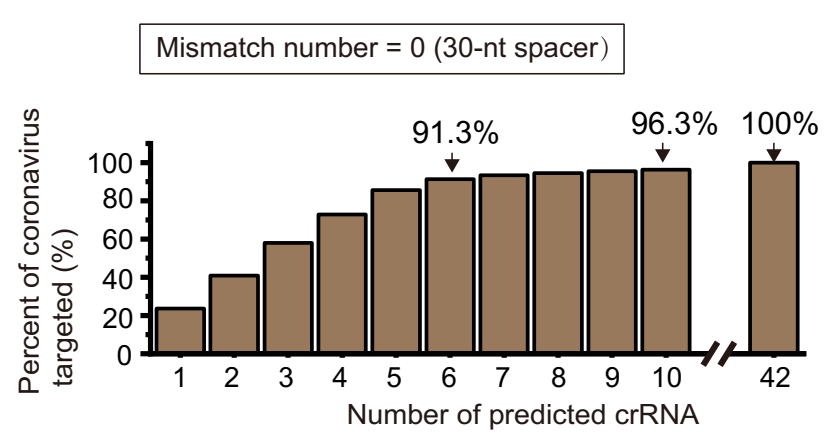

C

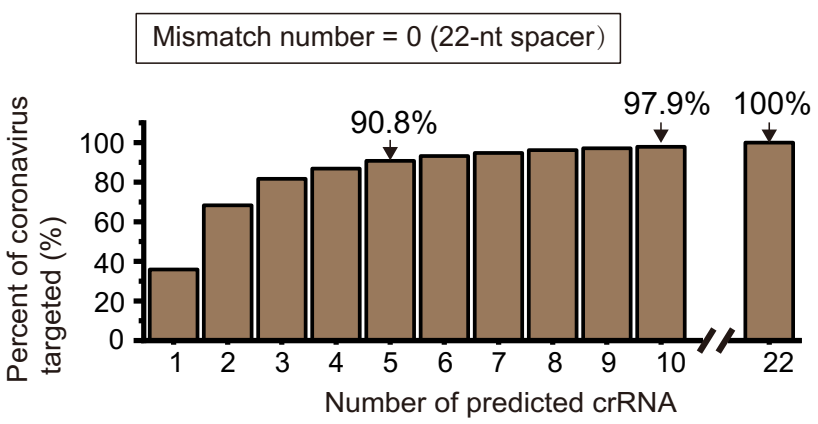

d

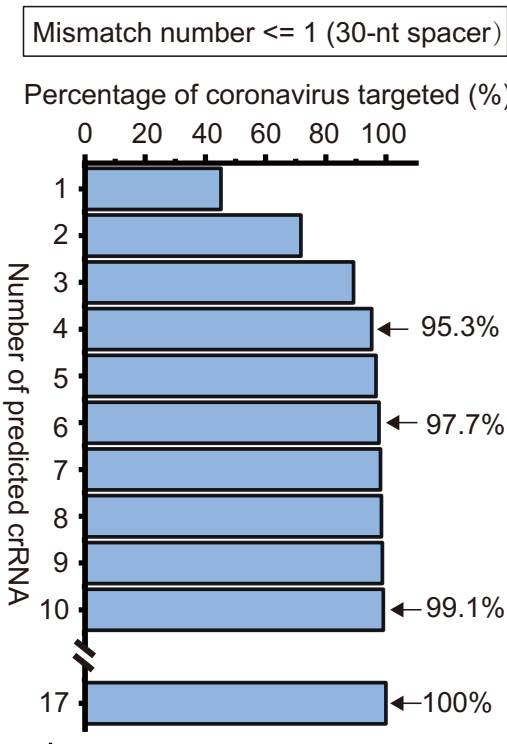

Spacers of minimized crRNA pool

ATCACACTTAGGATAATCCCAACCCATAAG
AGGGCTCTAGTCGAGTGCACTAGAGCCCC
CTGTACCCTCGACGTACTCCGCTGGCCT
AGGGTTCTAGTCGAGCTGCACTAGAACCCC
ATCACTCAAAATCATCATACTAAAATGCTT
GGTAACATAGTTCGTTTTCCAAATGTCCAA
TAACAATCCACATTACAATCCAAAACATA
GAATGCATGTTTATTAACATACAAACTACC
CACGGTGTAAGACGGGCTGCACTTACACCG
ATAAGCTGTGGTTGCATCACCACTGCTAGT
TACCACTAATAGCATACTTAAGATTAAGCT
GGCTCTAGTCGAGCACCACTAGAGCCCCGT
TACTAGACGGGCTACTCCACTAGTACCCCT
CATATTGTCCCAACCACCATAAAACTTGGT
ATTAGTCATACCCATAACAGGCATCATATA
TCACACTTAGGGTAATCCCAACCCATAAGA
TACCTGACAACACTCATTAGCCAAACGGTA

Genome Mismatch with numbers human transcriptome

$\begin{array}{ll}1416 & 5 \\ 848 & 6 \\ 1032 & 7 \\ 192 & 7 \\ 404 & 6 \\ 562 & 7 \\ 382 & 5 \\ 443 & 4 \\ 557 & 7 \\ 131 & 7 \\ 66 & 7 \\ 8 & 7 \\ 12 & 6 \\ 808 & 7 \\ 725 & 6 \\ 680 & 6 \\ 5 & 6\end{array}$

Extended Data Fig. 7 | Bioinformatics analysis for identifying minimal number of coronaviruses-targeting crRNAs. a, Schematic describing a computational pipeline for identifying the minimal number of crRNAs targeting all coronaviruses by analyzing available coronavirus genomes. b,c, Histograms showing the predicted minimal number of 22-nt and 30-nt crRNAs with zero mismatch to target all sequenced 3,137 coronavirus genomes. d, Seventeen 30-nt crRNAs with single-nt mismatch in the minimal pool that targets all coronaviruses and their similarity with human transcriptome. 
a

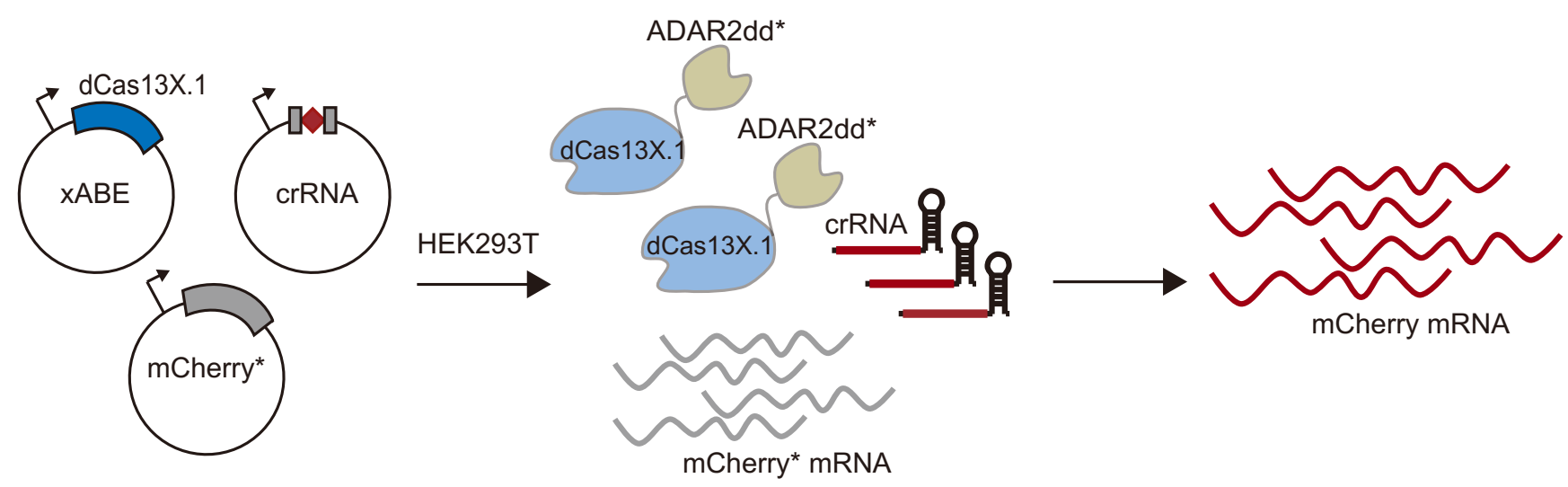

b

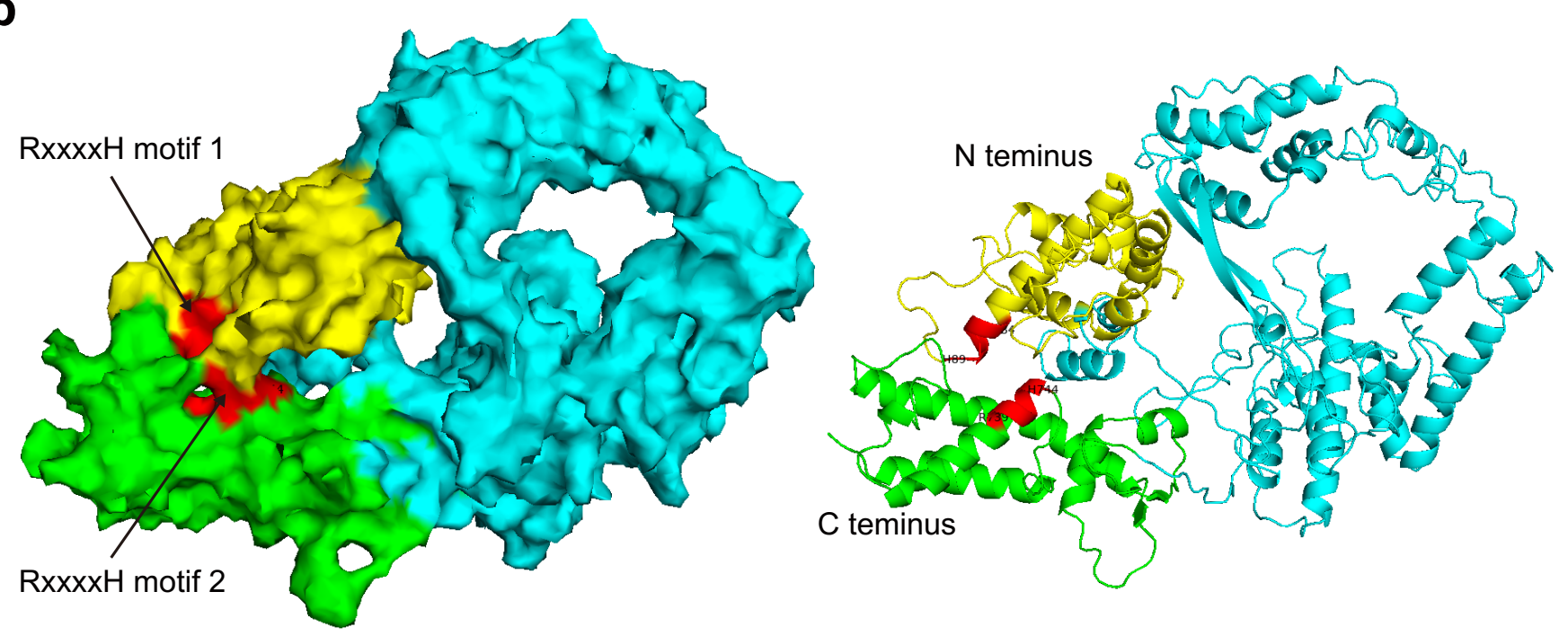

Extended Data Fig. 8 | Verification of eABE function with $\mathrm{mCherry}^{\star}$ reporter system and predicted Cas13X.1 protein structure. a, Schematic procedure of testing eABE with the $\mathrm{mCherry}^{\star}$ reporter system. $\mathbf{b}$, Predicted protein structure of Cas13X.1. Yellow denote $\mathrm{N}$ terminal; green indicate $\mathrm{C}$ terminal; red represent HEPN motif. 
a

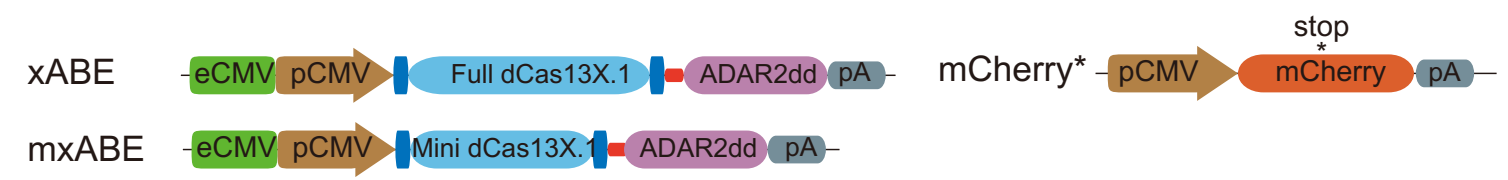

Target mRNA 5'-ACAUCCCCGACUACUUGAAGCUGUCCUUCCCCGAGGGCUUCAAGUAGGAGCGCGUGAUGAACUUCGAGGACGGCGGCGUGGUGACCGUGA-3' $\mathrm{CIII}_{3}$, UGUAGGGGCUGAUGAACUUCGACAGGAAGGGGCUCCCGAAGUUCACCCUC-5 CIII-GGGCUGAUGAACUUCGACAGGAAGGGGUCCCGAAGUUCACCCUCGCGCA-5' CIII-GAUGAACUUCGACAGGAAGGGGCUCCCGAAGUUCACCCUCGCGCACUACU-5'

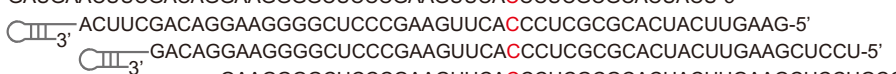
CIII, GAAGGGGCUCCCGAAGUUCACCCUCGCGCACUACUUGAAGCUCCUGCCGC-5' $\mathrm{CIII}_{3}$, GGCUCCCGAAGUUCACCCUCGCGCACUACUUGAAGCUCCUGCCGCCGCAC-5' $\mathrm{CI}_{3}$ 'CCGAAGUUCACCCUCGCGCACUACUUGAAGCUCCUGCCGCCGCACCACUG-5' ' ${ }^{\prime}{ }_{3}$ ' GUUCACCCUCGCGCACUACUUGAAGCUCCUGCCGCCGCACCACUGGCACU-5'

b

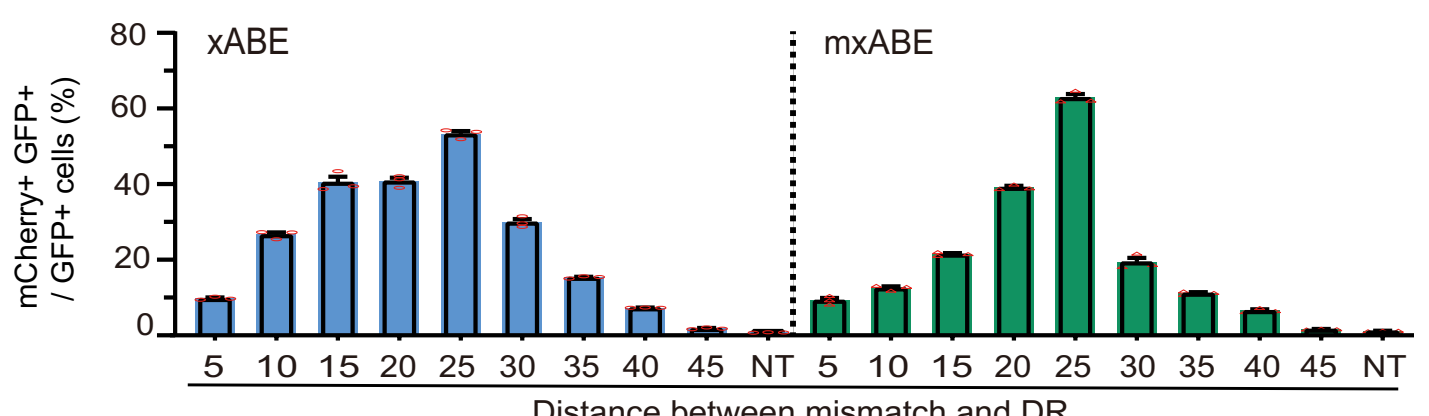

C

\begin{tabular}{|c|c|c|c|c|}
\hline crRNA & $\begin{array}{l}\text { A-to-I editing by } \\
\text { xABE A17 }\end{array}$ & $\begin{array}{l}\text { A-to-I editing by } \\
\text { mxABE A17 }\end{array}$ & $\begin{array}{l}\text { A-to-l editing by } \\
\text { mxABE A25 }\end{array}$ & Wild-type \\
\hline KRAS site 1 & 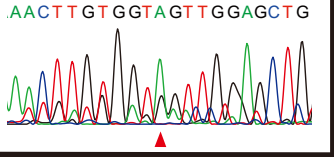 & 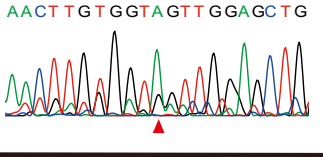 & alduld & 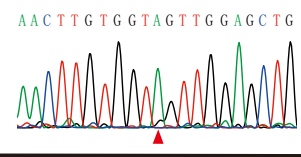 \\
\hline KRAS site2 & 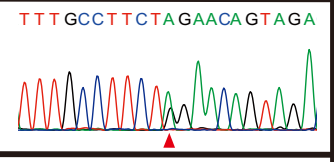 & 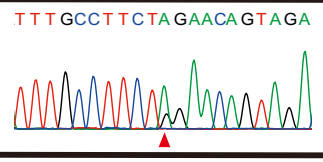 & TT TGCCT TCTAGAACA GTAGA & 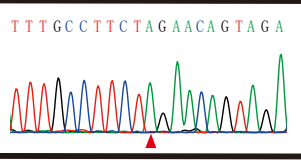 \\
\hline PPIB site1 & 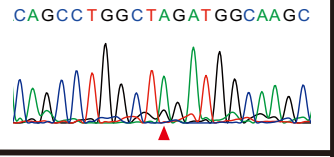 & $\frac{\text { cagcctagctagatgacaagc }}{\Delta}$ & 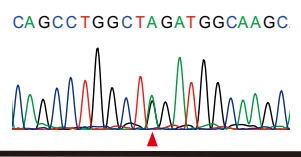 & 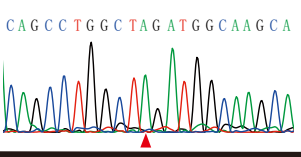 \\
\hline SMAD4 site1 & TCCAGC TCC TA GACGAAGTAC & 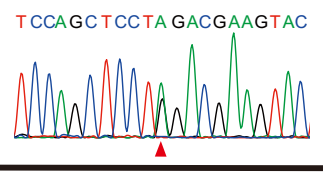 & TCCAGCTCCTAGACGAAGTAC & Whan \\
\hline SMAD4 site2 & Nam & Ala & Ala & 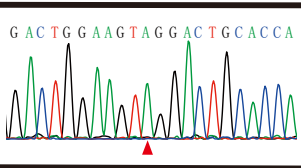 \\
\hline FANCC site 1 & 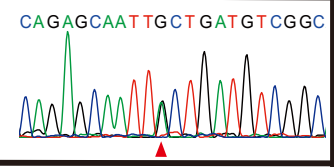 & 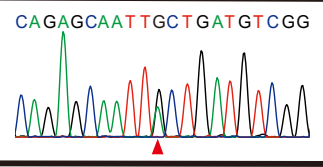 & 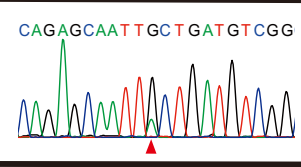 & $\frac{\text { MWhom }}{\Delta}$ \\
\hline FANCC site2 & 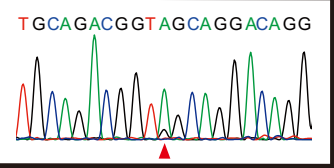 & 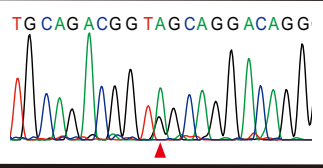 & 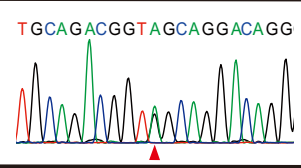 & Allw \\
\hline
\end{tabular}


Extended Data Fig. 9 | Effect of mismatched base position on xABE activity and Sanger sequencing results of editing human endogenous transcripts by $X A B E$. a, Mismatch positions in different crRNAs targeting the mCherry amber mutation. $\mathbf{b}$, Effect of mismatched base position with 50-nt spacer on A-to-I editing efficiency by both full and mini XABE editors. All values are presented as mean \pm s.e.m $(n=3)$. c, Sanger sequencing results showing representative A-to-I conversion on endogenous transcripts by full and mini XABE editors. Red triangles indicate mutation sites. 
a

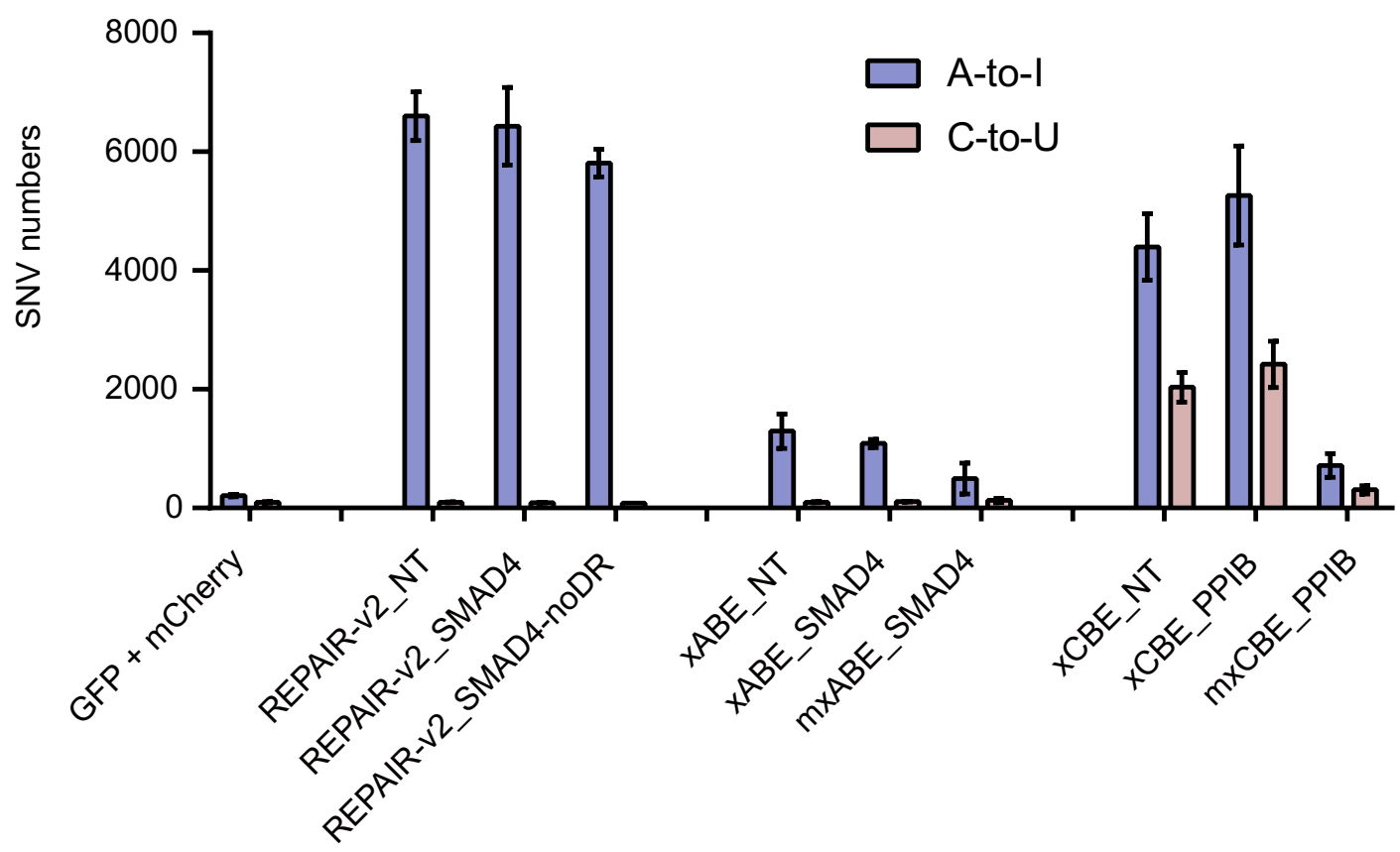

b

A to I

C to U

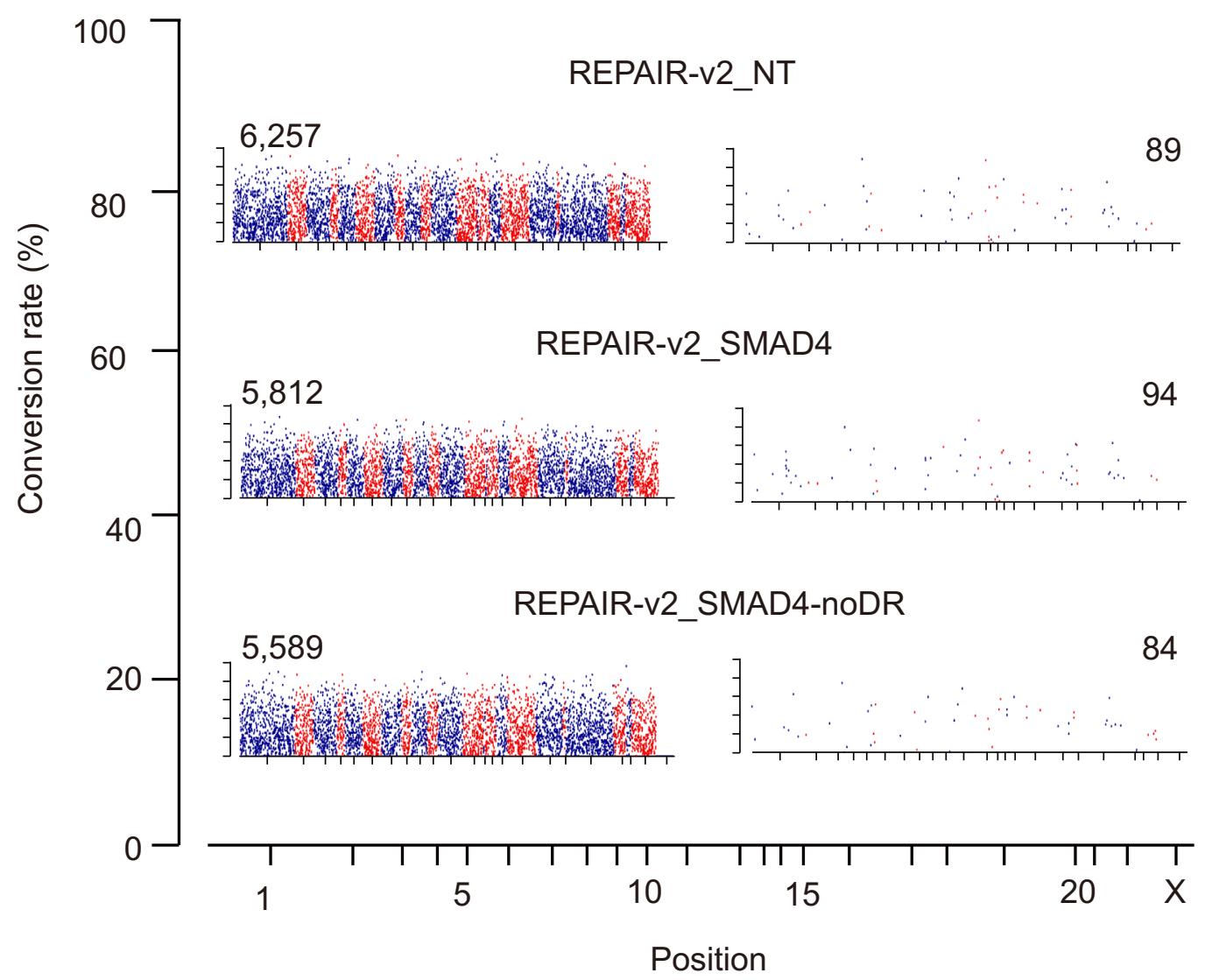

Extended Data Fig. 10 | See next page for caption. 
Extended Data Fig. 10 | Off-target RNA editing effect for xABE, mxABE, xCBE, mxCBE and REPAIR system. a, Transcriptome-wide off-target sites numbers for GFP/mCherry (control), xABE, $m \times A B E, x C B E$ and $m \times C B E$ transfection experiments in HEK293T cells. All values are presented as mean \pm s.e.m $(n=3)$. b. Manhattan plots of transcriptome-wide off-target RNA editing analysis for REPAIR transfection experiments in HEK293T cells (A-to-I editor targeting endogenous SMAD4 RNA). The $\mathrm{x}$ and $\mathrm{y}$ axis are proportionally enlarged with each Manhattan plot to make the axis legend clear. Non-DR, guide RNA without direct repeats. NT, non-targeting crRNA. 


\section{Reporting Summary}

Nature Research wishes to improve the reproducibility of the work that we publish. This form provides structure for consistency and transparency in reporting. For further information on Nature Research policies, see our Editorial Policies and the Editorial Policy Checklist.

\section{Statistics}

For all statistical analyses, confirm that the following items are present in the figure legend, table legend, main text, or Methods section.

n/a Confirmed

$\bigotimes$ The exact sample size $(n)$ for each experimental group/condition, given as a discrete number and unit of measurement

$\square$ A statement on whether measurements were taken from distinct samples or whether the same sample was measured repeatedly

The statistical test(s) used AND whether they are one- or two-sided

Only common tests should be described solely by name; describe more complex techniques in the Methods section.

Х $\square$ A description of all covariates tested

Х $\square$ A description of any assumptions or corrections, such as tests of normality and adjustment for multiple comparisons

$\triangle$ A full description of the statistical parameters including central tendency (e.g. means) or other basic estimates (e.g. regression coefficient)

AND variation (e.g. standard deviation) or associated estimates of uncertainty (e.g. confidence intervals)

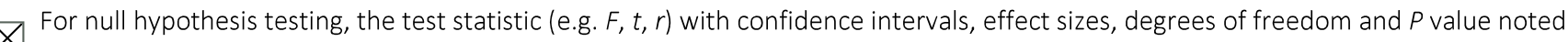
Give $P$ values as exact values whenever suitable.

Х $\square$ For Bayesian analysis, information on the choice of priors and Markov chain Monte Carlo settings

Х $\square$ For hierarchical and complex designs, identification of the appropriate level for tests and full reporting of outcomes

$\square$ Estimates of effect sizes (e.g. Cohen's d, Pearson's $r$ ), indicating how they were calculated

\section{Our web collection on statistics for biologists contains articles on many of the points above.}

\section{Software and code}

Policy information about availability of computer code

Data collection Next-generation sequencing data was collected and demultiplexed by lllumina HiSeq X-Ten platform. FACS data was generated using MoFlo XDP (Beckman Coulter) high-speed flow cytometry sorter.

Data analysis The custom Perl and Shell scripts of the computational pipeline used in this paper is available at https://github.com/yszhou2016/Cas13. Versions of softwares developed by other researchers were listed as follow.

SolexaQA (v3.I.7.1), Cutadapt(v2.8), Hisat2 (v2.0.4), htseq-count (v0.11.2), DEseq2 (v1.24.0), EditR(vl.0.1), CRISPResso2 (release 20180918), FlowJo X 10.0.7, MEGA(v7.0), Prodigal(v2.6.3), Piler-CR(vl.06), MAFFT (v7.464), I-TASSER(vS.I)

For manuscripts utilizing custom algorithms or software that are central to the research but not yet described in published literature, software must be made available to editors and

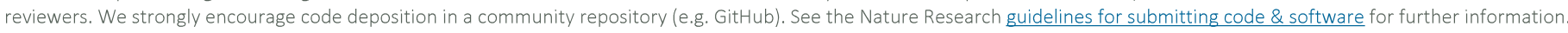

\section{Data}

Policy information about availability of data

All manuscripts must include a data availability statement. This statement should provide the following information, where applicable:

- Accession codes, unique identifiers, or web links for publicly available datasets

- A list of figures that have associated raw data

- A description of any restrictions on data availability

All sequencing datasets have been deposited in the NCBI SRA under project accession numbers PRJNA680488. dbSNP (v146) was downloaded from NCBI dbSNP databases. All other data supporting the findings of this study are available from the corresponding authors upon reasonable request. 
Please select the one below that is the best fit for your research. If you are not sure, read the appropriate sections before making your selection.

\ Life sciences

Behavioural \& social sciences

Ecological, evolutionary \& environmental sciences

For a reference copy of the document with all sections, see nature.com/documents/nr-reporting-summary-flat.pdf

\section{Life sciences study design}

All studies must disclose on these points even when the disclosure is negative.

$\begin{array}{ll}\text { Sample size } & \begin{array}{l}\text { No sample size calculation was performed. Based on previous publications, experiments in cell lines were performed in triplicates } n=3 \text {, unless } \\ \text { otherwise noted. }\end{array} \\ \text { Data exclusions } & \text { No data was excluded. } \\ \text { Replication } & \begin{array}{l}\text { We tested experimental conditions using different gRNAs to ensure robustness. We also performed at least three biological replicates for } \\ \text { each experiment and the experimental results could be successfully reproduced. }\end{array} \\ \text { Randomization } & \begin{array}{l}\text { Due to the small sample, randomization was not relevant for this study. Covariates were controlled for by running controls in parallel } \\ \text { whenever applicable. }\end{array} \\ \text { Blinding } & \begin{array}{l}\text { Blinding was not relevant to our study because in general, based on the prior experience of other groups in the field, these types of assays do } \\ \text { not require blinding. }\end{array}\end{array}$

\section{Reporting for specific materials, systems and methods}

We require information from authors about some types of materials, experimental systems and methods used in many studies. Here, indicate whether each material, system or method listed is relevant to your study. If you are not sure if a list item applies to your research, read the appropriate section before selecting a response.

\begin{tabular}{l|ll}
\multicolumn{2}{l}{ Materials \& experimental systems } & Methods \\
\hline $\mathrm{n} / \mathrm{a}$ & Involved in the study & \\
$\searrow$ & $\square$ Antibodies
\end{tabular}

\section{Eukaryotic cell lines}

Policy information about cell lines

Cell line source(s)

Authentication

Mycoplasma contamination

Commonly misidentified lines (See ICLAC register)
HEK293T and MDCK cells were obtained from Cell bank of Shanghai Institute of Biochemistry and eel I Biology, Chinese Academy of Sciences.

Cell lines were authenticated by the supplier.

Cell lines were tested and no contamination of mycoplasma.

None of the cell lines used was listed in the database of ICLAC. 
Plots

Confirm that:

\The axis labels state the marker and fluorochrome used (e.g. CD4-FITC).

$\bigotimes$ The axis scales are clearly visible. Include numbers along axes only for bottom left plot of group (a 'group' is an analysis of identical markers).

\All plots are contour plots with outliers or pseudocolor plots.

$\bigotimes$ A numerical value for number of cells or percentage (with statistics) is provided.

\section{Methodology}

Sample preparation

Instrument

Software

Cell population abundance

Gating strategy
To isolate cells, the transfected or non-transfected cells were dissociated enzymatically in an incubation solution of $5 \mathrm{ml}$ Trypsin-EDTA $(0.05 \%)$ at 37 "C for $5 \mathrm{~min}$. The digestion was stopped by adding $5 \mathrm{ml}$ of DMEM medium with $10 \%$ Fetal Bovine Serum (FBS). The cell suspension was centrifuged for $6 \mathrm{~min}(800 \mathrm{rpm})$, and the pellet was resuspended in DMEM medium with $10 \%$ FBS. Finally, the cell suspension was filtered through a 4(\} . $\mu \mathrm{m}$ cell strainer, and mCherry+, or GFP+ cells were isolated by FACS. Samples were found to be $>95 \%$ pure when assessed with a second round of flow cvtometrv and fluorescence microscopy analysis.

Cell Sorter (Beckman, MoFlo XDP)

FlowJoX 10.0.7

Samples were found to be $>95 \%$ pure when assessed with a second round of flow cvtometrv and fluorescence microscopy analysis.

Positive boundaries were determined by GFP+ or mCherry+ cells, and negative boundaries were determined by nontransfected cells.

\Tick this box to confirm that a figure exemplifying the gating strategy is provided in the Supplementary Information. 